Bull. Soc. math. France

131 (4), 2003, p. 483-506

\title{
IMAGE RÉCIPROQUE DU SQUELETTE PAR UN MORPHISME ENTRE ESPACES DE BERKOVICH DE MÊME DIMENSION
}

\author{
PAR Antoine Ducros
}

\begin{abstract}
RÉSumÉ. - Cet article concerne les espaces analytiques au sens de Berkovich. Soit $k$ un corps complet pour une valeur absolue ultramétrique et soit $\mathfrak{X}$ un schéma formel au-dessus de la boule unité $k^{0}$ de $k$. Si $\mathfrak{X}$ est pluristable (ce qui signifie essentiellement que les singularités de sa fibre spéciale sont « raisonnables ») alors sa fibre générique $\mathfrak{X}_{\eta}$ se rétracte sur l'un de ses sous-ensembles fermés noté $S(\mathfrak{X})$ (c'est le squelette de $\mathfrak{X}$ ) qui possède une structure naturelle d'espace linéaire par morceaux. Si $\mathfrak{Y} \rightarrow \mathfrak{X}$ est un morphisme étale entre deux schémas formels pluristables alors $S(\mathfrak{Y})$ est l'image réciproque de $S(\mathfrak{X})$, et $S(\mathfrak{Y}) \rightarrow S(\mathfrak{X})$ est linéaire par morceaux. Dans ce texte nous prouvons que si $\mathfrak{X}$ est pluristable purement de dimension $n$ et si $\varphi$ est un morphisme quelconque d'un espace strictement $k$-analytique topologiquement séparé de dimension $\leq n$ vers $\mathfrak{X}_{\eta}$ alors $\varphi^{-1}(S(\mathfrak{X}))$ possède une unique structure linéaire par morceaux telle que $\varphi$ soit linéaire par morceaux.
\end{abstract}

Texte reçu le 18 juin 2002, accepté le 10 décembre 2002

Antoine Ducros, IRMAR, Université de Rennes 1, Campus de Beaulieu, 35042 Rennes Cedex (France) • E-mail : antoine.ducros@univ-rennes1.fr

Classification mathématique par sujets (2000). — 14G22.

Mots clefs. - Espaces de Berkovich, structures linéaires par morceaux. 
Abstract (Pre-image of the skeleton under a map between Berkovich spaces of the same dimension)

This article deals with Berkovich analytic spaces. Let $k$ be a complete field with respect to an ultrametric absolute value and let $\mathfrak{X}$ be a formal scheme over the unit ball $k^{0}$ of $k$. If $\mathfrak{X}$ is pluri-stable (roughly speaking, it means that the singularities of its special fibre are "not too bad") then its generic fibre $\mathfrak{X}_{\eta}$ admits a retraction toward a closed subset $S(\mathfrak{X})$ (the skeleton of $\mathfrak{X}$ ) which carries a natural structure of piecewiselinear space. If $\mathfrak{Y} \rightarrow \mathfrak{X}$ is an étale morphism between two pluri-stable formal schemes then $S(\mathfrak{Y})$ is exactly the pre-image of $S(\mathfrak{X})$, and $S(\mathfrak{Y}) \rightarrow S(\mathfrak{X})$ is piecewise-linear. Here we show that if $\mathfrak{X}$ is pluri-stable of pure dimension $n$ and if $\varphi$ is any morphism from an Hausdorff strictly $k$-analytic space of dimension $\leq n$ to $\mathfrak{X}_{\eta}$ then $\varphi^{-1}(S(\mathfrak{X}))$ carries a unique piecewise-linear structure such that $\varphi$ is piecewise-linear.

\section{Introduction}

Soient $k$ un corps complet pour une valeur absolue ultramétrique $|$.$| et k^{0}$ le sous-anneau de $k$ formé des éléments de valeur absolue inférieure ou égale à 1. Vladimir Berkovich a développé dans [2] et [3] une théorie des espaces $k$-analytiques qui est reliée à la théorie rigide classique mais en diffère notamment par le fait que les espaces qu'il construit possèdent de bonnes propriétés topologiques : ainsi, chacun de leurs points a une base de voisinages compacts et connexes par arcs.

Berkovich a défini (voir [4], §1), d'une manière analogue à ce qui se fait dans le cadre rigide, un foncteur fibre générique qui à tout $k^{0}$-schéma formel $\mathfrak{X}$ satisfaisant des hypothèses de finitude raisonnables associe un espace $k$-analytique $\mathfrak{X}_{\eta}$. Soit $\mathfrak{X}$ un $k^{0}$-schéma formel pluristable, c'est-à-dire tel que le morphisme structural $\mathfrak{X} \rightarrow \operatorname{Spf} k^{0}$ soit localement isomorphe pour la topologie étale à une composition de flèches qui sont ou bien étales ou bien de la forme

$$
\operatorname{Spf}\left(\mathfrak{A}\left\{T_{1}, \ldots, T_{n}\right\} /\left(T_{1} \ldots T_{n}-a\right)\right) \longrightarrow \operatorname{Spf} \mathfrak{A} .
$$

Berkovich a alors démontré (voir [5], th. 8.1 et [1], §4.4) que $\mathfrak{X}_{\eta}$ se rétracte sur l'un de ses sous-ensembles fermés noté $S(\mathfrak{X})$ et appelé le squelette de $\mathfrak{X}$, sous-ensemble qui est un complexe cellulaire et possède, si de plus l'espace $k$ analytique $\mathfrak{X}_{\eta}$ est normal, une structure d'espace linéaire par morceaux (voir [1], $\S \S 4$ et 5$)$.

Le squelette se comporte de manière fonctorielle relativement aux morphismes étales de schémas formels pluristables à fibre générique normale; plus précisément si $\varphi: \mathfrak{Y} \rightarrow \mathfrak{X}$ est un tel morphisme, alors $S(\mathfrak{Y})$ est l'image réciproque de $S(\mathfrak{X})$ par la flèche $\varphi_{\eta}: \mathfrak{Y}_{\eta} \rightarrow \mathfrak{X}_{\eta}$ et $S(\mathfrak{Y}) \rightarrow S(\mathfrak{X})$ est linéaire par morceaux.

Le but de cet article est d'étudier l'image réciproque du squelette par un morphisme quelconque entre espaces de Berkovich de même dimension. On établit plus précisément le théorème suivant (théorème 3.1) : 
ThÉORÈme. - Soit $k$ un corps valué complet. Soient $n$ un entier et $\mathfrak{X}$ un $k^{0}$-schéma formel pluristable non dégénéré purement de dimension $n$. Soit $Z$ un espace strictement $k$-analytique topologiquement séparé de dimension inférieure ou égale à $n$ et $\varphi$ un morphisme de $Z$ vers $\mathfrak{X}_{\eta}$. Posons $\Delta=\varphi^{-1}(S(\mathfrak{X}))$. Il existe alors une unique structure linéaire par morceaux sur $\Delta$ telle que $\varphi_{\mid \Delta}: \Delta \rightarrow S(\mathfrak{X})$ soit linéaire par morceaux, et $\varphi_{\mid \Delta}$ est $G$-localement une immersion.

L'une des motivations de ce travail réside dans l'espoir (encore bien lointain à ce jour) d'obtenir une généralisation en dimension supérieure d'un résultat [8] de l'auteur qui concerne les courbes algébriques $p$-adiques; une telle généralisation supposerait déjà d'établir l'analogue de plusieurs propositions techniques intermédiaires de [8], comme la « variation du corps résiduel » (cf. [8], prop. 2.3) ou la notion de « polyèdre de variation » ( $c f .[8]$, prop. 1.21). Le théorème démontré ici a justement comme corollaires certains des résulats souhaités ( $c f . \S 3.29$ et 3.30$)$.

\section{Préliminaires et notations}

0.1. On appellera corps valué un corps muni d'une valeur absolue ultramétrique non triviale. Si $k$ est un tel corps on emploiera, sauf mention expresse du contraire, les notations suivantes :

- |. | pour la valeur absolue de $k$.

- $k^{0}$ pour le sous-anneau $\{x \in k$ t.q. $|x| \leq 1\}$ de $k$ et $k^{00}$ pour l'idéal de $k^{0}$ formé des éléments de valeur absolue strictement inférieure à 1 . Le quotient $k^{0} / k^{00}$ sera noté $\widetilde{k}$ et appelé corps résiduel de $k$.

- $\sqrt{\left|k^{*}\right|}$ pour le sous-groupe de $\mathbb{R}_{+}^{*}$ formé des éléments de torsion modulo $\left|k^{*}\right|$.

0.2. Soit $k$ un corps. On appellera $k$-variété algébrique tout $k$-schéma séparé de type fini. Pour tout entier $n$, on notera $\mathbb{A}_{k}^{n}$ l'espace affine de dimension $n$ sur $k$. On notera indifféremment $\mathbb{G}_{m, k}$ le groupe multiplicatif et la $k$-variété $\mathbb{A}_{k}^{1}-\{0\}$ qui lui est sous-jacente.

On fixe un corps valué complet $k$.

0.3. Le terme d'espace $k$-analytique sera dans la suite à prendre au sens de Berkovich, et plus précisément de [3] ; les notions de base de sa théorie, qui sont pour l'essentiel définies dans le chapitre 1 de [3], seront utilisées sans rappel ni justification. Un espace $k$-analytique est en particulier un espace topologique au sens classique. De plus, la catégorie de ses domaines analytiques peut être munie d'une topologie de Grothendieck que l'on appellera la $G$-topologie. Si $\mathcal{X}$ est une $k$-variété algébrique on notera $\mathcal{X}^{\text {an }}$ son analytification. Si $\mathcal{A}$ est une algèbre $k$-affinoïde on notera $\mathcal{M}(\mathcal{A})$ l'espace $k$-analytique qui lui est associé. 
Si $X$ est un espace $k$-analytique et $P$ un point de $X$ on notera $\mathcal{H}(P)$ le corps résiduel complété de $P$. Notons que ce corps est invariant par immersion, ce qui justifie l'omission de $X$ dans la notation $\mathcal{H}(P)$. Un espace $k$-analytique sera dit intègre s'il est irréductible et réduit.

0.4. On dira qu'un espace $k$-analytique est algébroïde s'il est isomorphe à un domaine analytique de l'analytification $\mathcal{X}^{\text {an }}$ d'une $k$-variété algébrique $\mathcal{X}$.

0.5. Si $X$ est algébroïde et si $Y \rightarrow X$ est quasi-étale (cette notion est définie au paragraphe 3 de [4]), on déduit du lemme de Krasner et du théorème 3.4.1 de [3] que tout point de $Y$ possède un voisinage algébroïde.

0.6. Soit $\mathcal{X}$ un $k^{0}$-schéma. On notera $\mathcal{X}_{\eta}$ sa fibre générique, $\mathcal{X}_{s}$ sa fibre spéciale et $\widehat{\mathcal{X}}$ le complété formel de $\mathcal{X}$ le long de $\mathcal{X}_{s}$. Si $\mathfrak{X}$ est un $k^{0}$-schéma formel, on notera $\mathfrak{X}_{s}$ sa fibre spéciale; si $\mathfrak{X}$ admet un recouvrement localement fini par des ouverts de présentation finie sur $\operatorname{Spf} k^{0}$, on peut définir $(c f .[4], \S 1)$ sa fibre générique $\mathfrak{X}_{\eta}$ qui est un $k$-espace analytique. On dispose alors d'une application de réduction $\pi: \mathfrak{X}_{\eta} \rightarrow \mathfrak{X}_{s}$.

Si $\mathcal{X}$ est un $k^{0}$-schéma quelconque, on a $\mathcal{X}_{s}=\widehat{\mathcal{X}}_{s}$; s'il admet un recouvrement localement fini par des ouverts de présentation finie sur Spec $k^{0}$, on dispose d'une flèche naturelle $\widehat{\mathcal{X}}_{\eta} \rightarrow \mathcal{X}_{\eta}^{\text {an }}$ qui est un isomorphisme si $\mathcal{X}$ est propre $(c f .[4], \S 5)$.

0.7. Dans tout le texte les définitions, notations et conventions relatives aux espaces linéaires par morceaux sont celles introduites par Berkovich dans [1], $\S 1$. Le terme « linéaire par morceaux » sans plus de précision signifiera toujours dans la suite $《 \sqrt{\left|k^{*}\right|_{\mathbb{Q}}}$-linéaire par morceaux». Tout espace linéaire par morceaux est muni d'une topologie (au sens classique). On peut par ailleurs définir, sur la catégorie de ses sous-espaces linéaires par morceaux, une topologie de Grothendieck que l'on appellera simplement la $G$-topologie. Notons que comme $\mathbb{Q}$ est un corps et $\sqrt{\left|k^{*}\right|}$ un $\mathbb{Q}$-espace vectoriel toute application linéaire par morceaux bijective entre espaces linéaires par morceaux est un isomorphisme (ce qui est faux pour des ensembles de coefficients plus généraux).

0.8. Soit $\mathfrak{X}$ un $k^{0}$-schéma formel pluristable non dégénéré ( $c f$. [5], $\S 1$ et [1], $\S 4.1)$. On notera $S(\mathfrak{X})$ son squelette; c'est un sous-ensemble fermé de $\mathfrak{X}_{\eta}$ sur lequel ce dernier se rétracte ( $c f .[1], \S \S 4$ et 5 ), et qui peut être muni d'une structure $\left(\left|k^{*}\right| \cap[0 ; 1]\right)_{\mathbb{Z}_{+}}$-linéaire par morceaux. On peut en particulier le voir comme un espace linéaire par morceaux au sens donné au $\S 0.7$ ci-dessus, ce que l'on fera désormais.

0.9. Soit $\ell$ un entier naturel. On dira qu'un $k^{0}$-schéma formel $\mathfrak{X}$ admet une bonne fibration de longueur $\ell$ si le morphisme structural $\mathfrak{X} \rightarrow \operatorname{Spf} k^{0}$ peut se décomposer sous la forme

$$
\mathfrak{X}=\mathfrak{X}_{\ell} \rightarrow \mathfrak{Z}_{\ell} \rightarrow \mathfrak{X}_{\ell-1} \rightarrow \mathfrak{Z}_{\ell-1} \rightarrow \mathfrak{X}_{\ell-2} \rightarrow \cdots \rightarrow \mathfrak{Z}_{0}=\operatorname{Spf} k^{0}
$$

TOME $131-2003-\mathrm{N}^{\mathrm{O}} 4$ 
où :

- pour tout $j$ compris entre 0 et $\ell$, les schémas formels $\mathfrak{X}_{j}$ et $\mathfrak{Z}_{j}$ sont affines;

- pour tout $j$ compris entre 0 et $\ell$, la flèche $\mathfrak{X}_{j} \rightarrow \mathfrak{Z}_{j}$ est étale et induit un isomorphisme entre les ensembles polysimpliciaux associés, et donc entre $S\left(\mathfrak{X}_{j}\right)$ et $S\left(\mathfrak{Z}_{j}\right)$;

- pour tout $j$ compris entre 1 et $\ell$, le $\mathfrak{X}_{j-1}$-schéma formel $\mathfrak{Z}_{j}$ est isomorphe à $\mathfrak{X}_{j-1}\left(\mathbf{n}_{j}, \mathbf{a}_{j}, m_{j}\right)$ pour une famille $\left(\mathbf{n}_{j}, \mathbf{a}_{j}, m_{j}\right)$ convenable (pour le sens de cette notation, $c f .[1], \S 4.1)$ telle que les fonctions sur $\mathfrak{X}_{j-1}$ qui constituent la famille $\mathbf{a}_{j}$ soient toutes non nulles.

Notons que si un $k^{0}$-schéma formel admet une bonne fibration, il est strictement pluristable non dégénéré; sa fibre générique est irréductible.

0.10. Si $\left(r_{1}, \ldots, r_{n}\right)$ est une famille de $n$ réels strictement positifs, on notera $\eta_{k}^{r_{1}, \ldots, r_{n}}$ le point de $\mathbb{A}_{k}^{n, \text { an }}$ défini par la semi-norme

$$
\sum a_{i_{1}, \ldots, i_{n}} T_{1}^{i_{1}} \cdots T_{n}^{i_{n}} \longmapsto \max \left|a_{i_{1}, \ldots, i_{n}}\right| r_{1}^{i_{1}} \cdots r_{n}^{i_{n}} .
$$

L'application $\left(r_{1}, \ldots, r_{n}\right) \mapsto \eta_{k}^{r_{1}, \ldots, r_{n}}$ induit un homéomorphisme entre $\left(\mathbb{R}_{+}^{*}\right)^{n}$ et un sous-ensemble $S_{k}^{n}$ de $\mathbb{A}_{k}^{n \text {,an }}$, qui est fermé dans l'ouvert $\left(\mathbb{G}_{m, k}^{\text {an }}\right)^{n}$ de $\mathbb{A}_{k}^{n \text {,an }}$.

0.11. Remarque. - Si $L / k$ est une extension finie de corps valués, alors $S_{L}^{n}$ est l'image réciproque de $S_{k}^{n}$ par la flèche naturelle $\mathbb{A}_{L}^{n \text {,an }} \rightarrow \mathbb{A}_{k}^{n \text {,an }}$ et l'application continue $S_{L}^{n} \rightarrow S_{k}^{n}$ ainsi induite est un homéomorphisme.

0.12. Pour toute famille $\left(r_{1}, \ldots, r_{n}\right)$ de réels strictement positifs, le corps résiduel complété $\mathcal{H}\left(\eta_{k}^{r_{1}, \ldots, r_{n}}\right)$ sera noté $k_{r_{1}, \ldots, r_{n}}$. Si la famille des $r_{i}$ est libre dans $\left(\mathbb{R}_{+}^{*} /\left|k^{*}\right|\right) \otimes_{\mathbb{Z}} \mathbb{Q}$, alors $k_{r_{1}, \ldots, r_{n}}$ est l'ensemble des séries formelles

$$
\sum a_{i_{1}, \ldots, i_{n}} T_{1}^{i_{1}} \cdots T_{n}^{i_{n}}
$$

où les $i_{j}$ appartiennent à $\mathbb{Z}$ et telles que $\left|a_{i_{1}, \ldots, i_{n}}\right| r_{1}^{i_{1}} \cdots r_{n}^{i_{n}}$ tende vers zéro lorsque $\left|i_{1}\right|+\cdots+\left|i_{n}\right|$ tend vers l'infini. La valeur absolue d'une telle série est égale à $\max \left|a_{i_{1}, \ldots, i_{n}}\right| r_{1}^{i_{1}} \cdots r_{n}^{i_{n}}$.

0.13. Par analogie avec le cas des schémas on dira qu'un morphisme $Y \rightarrow X$ d'espaces $k$-analytiques est radiciel s'il est universellement injectif, c'est-à-dire s'il est injectif et si $\mathcal{H}(P)$ est une extension radicielle de $\mathcal{H}(\varphi(P))$ pour tout point $P$ de $X$. Un revêtement fini radiciel et plat induit un homéomorphisme entre les espaces topologiques sous-jacents.

0.14. Soient $\mathcal{A}$ une algèbre $k$-affinoïde intègre et $\mathcal{B}$ une $\mathcal{A}$-algèbre intègre finie et plate, telle que la flèche $\mathcal{M}(\mathcal{B}) \rightarrow \mathcal{M}(\mathcal{A})$ soit radicielle. Le corps des fractions de $\mathcal{B}$ est alors une extension finie purement inséparable de celui de $\mathcal{A}$. On en déduit l'existence d'un entier $r$ strictement positif (qui est une puissance de la caractéristique de $k$ ) tel que pour tout élément $f$ de $\mathcal{B}$ la fonction $f^{r}$ provienne du corps des fractions de $\mathcal{A}$; par fidèle platitude on conclut que $f^{r}$ provient de 
$\mathcal{A}$ (appliquer la prop. 9c) de [7], chap. I, §3, en prenant pour $\mathcal{A}$-module $F$ le quotient de $\mathcal{A}$ par un dénominateur de $f^{r}$ ).

\section{Le rang total d'une extension de corps valués}

L'auteur a introduit la notion de rang total et établi ses premières propriétés pour les besoins de cet article. En fait Berkovich avait déjà considéré certaines de ces questions au chapitre 9 de [2]; le rang total ici défini correspond à ce qu'il note $d(K)$ et le lemme 1.6 à sa proposition 9.1.3.

1.1. DÉfinition. - Soit $L / k$ une extension de corps valués. On définit son rang total comme l'élément

$$
\operatorname{deg} \operatorname{tr}(\widetilde{L} / \widetilde{k})+\operatorname{dim}_{\mathbb{Q}}\left(\left(\left|L^{*}\right| /\left|k^{*}\right|\right) \otimes_{\mathbb{Z}} \mathbb{Q}\right)
$$

de $\mathbb{N} \cup\{+\infty\}$. On le note $\rho(L / k)$.

1.2. Si $M / L$ et $L / k$ sont deux extensions de corps valués alors

$$
\rho(M / k)=\rho(M / L)+\rho(L / k) .
$$

1.3. Exemple. - Soit $k$ un corps valué, soit $t$ une indéterminée et $r$ un réel strictement positif. Munissons $k(t)$ de la valeur absolue $\sum a_{i} t^{i} \mapsto \max \left|a_{i}\right| r^{i}$. Si $r$ est de torsion modulo $\left|k^{*}\right|$, alors $\left|k(t)^{*}\right| /\left|k^{*}\right|$ est fini et $\widetilde{k(t)}$ est transcendant pur de degré 1 sur $\widetilde{k}$. Dans le cas contraire $\left|k(t)^{*}\right| /|k(t)|$ est libre de rang 1 et $\widetilde{k(t)}$ est égal à $\widetilde{k}$. Dans les deux cas $\rho(k(t) / k)=1$.

1.4. Exemple. - Soit $k$ un corps valué complet et $\left(r_{1}, \ldots, r_{n}\right)$ une famille de $n$ réels strictement positifs. Considérons $k\left(T_{1}, \ldots, T_{n}\right)$ comme un sous-corps valué de $k_{r_{1}, \ldots, r_{n}}$. On cherche à déterminer $\rho\left(k\left(T_{1}, \ldots, T_{n}\right) / k\right)$. L'exemple 1.3 ci-dessus, combinée au $\S 1.2$ et à une récurrence immédiate, entraîne l'égalité $\rho\left(k\left(T_{1}, \ldots, T_{n}\right) / k\right)=n$. Comme $k\left(T_{1}, \ldots, T_{n}\right)$ est dense dans $k_{r_{1}, \ldots, r_{n}}$, on a aussi $\rho\left(k_{r_{1}, \ldots, r_{n}} / k\right)=n$.

1.5. Lemme. - Soit $n$ un entier et soit $\mathfrak{X}$ un $k^{0}$-schéma formel pluristable non dégénéré purement de dimension $n$. Soit $P$ appartenant à $S(\mathfrak{X})$. Alors $\rho(\mathcal{H}(P) / k)=n$.

Démonstration. - Un morphisme étale de schémas formels induit un morphisme quasi-étale entre leurs fibres génériques (cf. [4], prop. 2.3); et un morphisme quasi-étale conserve le rang total du corps résiduel d'un point au-dessus de $k$ (puisque le rang total d'une extension finie est nul).

On sait par Berkovich [1], 55.5 , que tout $k^{0}$-schéma formel pluristable non dégénéré $\mathfrak{X}$ possède un recouvrement étale par une famille $\left(\mathfrak{X}_{i}\right)$ où $\mathfrak{X}_{i}$ est pour tout $i$ un $k^{0}$-schéma formel admettant une bonne fibration. La combinaison de cette observation et de la remarque ci-dessus permet de se ramener au cas où $\mathfrak{X}$ admet une bonne fibration d'une certaine longueur $\ell$. On conclut en raisonnant 
par récurrence $\operatorname{sur} \ell$. Le cas $\ell=0$ est trivial. Le passage du rang $\ell$ au rang $\ell+1$ repose sur une description explicite du squelette des fibres de $\mathfrak{Z}_{\ell, \eta} \rightarrow \mathfrak{X}_{\ell-1, \eta}$ (fondée sur les résultats de Berkovich établis dans [5], étape 3 de la preuve du th. 5.2), sur l'exemple 1.4 et sur la remarque faite en début de preuve pour traiter le passage de $\mathfrak{Z}_{\ell}$ à $\mathfrak{X}_{\ell}$.

Le lemme qui suit a été antérieurement établi par Berkovich (c'est la proposition 9.1.3 de [2], avec la même preuve que ci-dessous). Nous en donnons la démonstration pour la commodité du lecteur.

1.6. Lemme. - Soient $k$ un corps valué complet et $X$ un espace strictement $k$-analytique. Alors

$$
\operatorname{dim} X=\max _{P \in X} \rho(\mathcal{H}(P) / k) .
$$

Démonstration. - La dimension de $X$ étant égale à la dimension maximale d'un domaine affinoïde de $X$, on se ramène aussitôt au cas où $X$ lui-même est strictement $k$-affinoïde. Le lemme de normalisation de Nother (cf. [6], cor. 2, p. 228) permet ensuite de supposer que $X$ est le polydisque unité de dimension $n$. On conclut par une récurrence facile sur $n$ fondée sur la description explicite des points de la droite projective (voir [2], 1.4.4 et [3], §3.6).

1.7. Remarque. - On peut montrer que l'assertion reste vraie pour un espace $k$-analytique quelconque (on se ramène au cas strictement $k$-analytique par changement de corps), mais nous n'en aurons pas besoin ici.

1.8. Lemme. - Soit $k$ un corps valué complet et soit $n$ un entier. Soit $\mathcal{A}$ une algèbre strictement $k$-affinoïde réduite de dimension $n$ et $\mathcal{B}$ une $\mathcal{A}$-algèbre finie. Notons $\varphi$ la flèche $\mathcal{M}(\mathcal{B}) \rightarrow \mathcal{M}(\mathcal{A})$. Soit $x$ un point de $\mathcal{M}(\mathcal{A})$ possédant un et un seul antécédent y par $\varphi$ et tel que $\rho(\mathcal{H}(x) / k)=n$; faisons l'hypothèse que $\mathcal{H}(y) / \mathcal{H}(x)$ est purement inséparable. Il existe alors un ouvert Zariski $\Omega$ de $\mathcal{M}(\mathcal{A})$ contenant $x$ tel que $\varphi^{-1}(\Omega) \rightarrow \Omega$ soit fini, radiciel et plat.

Démonstration. - Désignons par $\varpi$ la flèche naturelle $\mathcal{M}(\mathcal{A}) \rightarrow \operatorname{Spec} \mathcal{A}$. Comme $\rho(\mathcal{H}(x) / k)=n$, le lemme 1.6 ci-dessus montre que $x$ n'appartient à aucun sous-ensemble fermé analytique de $\mathcal{M}(\mathcal{A})$ de dimension strictement inférieure à $n$. En conséquence $\varpi(x)$ est le point générique $\eta$ de l'une des composantes irréductibles de $\operatorname{Spec} \mathcal{A}$. La fibre $\operatorname{de} \operatorname{Spec} \mathcal{B} \rightarrow \operatorname{Spec} \mathcal{A}$ au-dessus de $\eta$ est nécessairement réduite à un point dont le corps résiduel est une extension finie purement inséparable du corps résiduel de $\eta$. Ceci entraîne, l'anneau $\mathcal{A}$ étant réduit, l'existence d'un ouvert Zariski $\mathcal{U} \operatorname{de} \operatorname{Spec} \mathcal{A}$ contenant $\eta$ tel que $\operatorname{Spec} \mathcal{B} \times \operatorname{Spec} \mathcal{A} \mathcal{U} \rightarrow \mathcal{U}$ soit fini, radiciel et plat. On en déduit déjà, à l'aide de la prop. 3.2.1 de [3], que $\mathcal{M}(\mathcal{B}) \rightarrow \mathcal{M}(\mathcal{A})$ est fini et plat au-dessus de $\varpi^{-1}(\mathcal{U})$. Montrons qu'il est radiciel au-dessus de ce même ouvert. Cela résulte du simple fait suivant : si $E / F$ est une extension finie purement inséparable de corps et si $|$.$| est une valeur absolue ultramétrique sur F$, alors $|$.$| admet une$ 
unique extension à $E$ et l'extension correspondante $\widehat{E} / \widehat{F}$ entre les complétés est purement inséparable. La démonstration est terminée.

1.9. Lemme. - Soit $k$ un corps valué complet, soit $n$ un entier et soit $Y$ un espace strictement $k$-analytique de dimension inférieure ou égale à $n$. Soit $\varphi$ un morphisme de $Y$ vers un bon espace strictement $k$-analytique $X$ et soit $P$ un point de $Y$ tel que $\rho(\mathcal{H}(\varphi(P)) / k)=n$. Sous ces hypothèses :

(i) la fibre $\varphi^{-1}(\varphi(P))$ est discrète;

(ii) si $P$ appartient à Int $Y / X$, alors $\varphi$ est fini en $P$;

(iii) si $P$ appartient à Int $Y / X$ et si de plus $X$ est réduit et de dimension $n$, il existe un voisinage strictement $k$-affinoïde $U$ de $P$ dans $Y$ tel que la restriction de $\varphi$ à $U$ se factorise sous la forme

$$
U \longrightarrow W \longrightarrow V \longrightarrow X
$$

où

- $V \rightarrow X$ identifie $V$ à un voisinage strictement $k$-affinoïde de $\varphi(P)$ dans $X$,

- $W \rightarrow V$ est un revêtement fini étale,

- $U \rightarrow W$ est un revêtement fini radiciel et plat.

Démonstration. - Soit $Q$ appartenant à $\varphi^{-1}(\varphi(P))$. On a l'égalité

$\rho(\mathcal{H}(Q) / k)=\rho(\mathcal{H}(Q) / \mathcal{H}(\varphi(P)))+\rho(\mathcal{H}(\varphi(P)) / k)=\rho(\mathcal{H}(Q) / \mathcal{H}(\varphi(P)))+n$.

Comme $Y$ est de dimension inférieure ou égale à $n$, le lemme 1.6 implique que $\rho(\mathcal{H}(Q) / k) \leq n$. On en déduit que $\rho(\mathcal{H}(Q) / \mathcal{H}(\varphi(P)))=0$, et ce pour tout point $Q$ appartenant à l'espace $\mathcal{H}(\varphi(P))$-analytique $\varphi^{-1}(\varphi(P))$. Ce dernier est donc, toujours d'après le lemme 1.6 , de dimension nulle, et donc est discret ce qui montre (i). En particulier $P$ est isolé dans sa fibre et donc si $P$ appartient à Int $Y / X$ le morphisme $\varphi$ est fini en $P$ en vertu de la prop. 3.1 .4 c) de [3], ce qui montre (ii); notons que sous ces hypothèses il existe un voisinage strictement $k$-affinoïde $U$ de $P$ dans $Y$ et un voisinage strictement $k$-affinoïde $V$ de $\varphi(P)$ dans $X$ tels que $\varphi$ induise un morphisme fini de $U$ dans $V$.

Montrons maintenant (iii). Soit $L$ la clôture séparable de $\mathcal{H}(\varphi(P))$ dans $\mathcal{H}(P)$. La catégorie des $\mathcal{H}(\varphi(P))$-algèbres finies étales est équivalente à celle des revêtements finis étales du germe $(X, \varphi(P))$ ( $c f$. [3], th. 3.4.1). Quitte à restreindre $V$ et $U$, on peut donc supposer qu'il existe un revêtement fini étale $W \rightarrow V$ dont la fibre en $\varphi(P)$ est isomorphe à $\mathcal{M}(L)$. Dès lors la fibre en $P$ du revêtement étale $W \times_{V} U \rightarrow U$ possède un point $P^{\prime}$ tel que l'injection $\mathcal{H}(P) \hookrightarrow \mathcal{H}\left(P^{\prime}\right)$ soit un isomorphisme; en conséquence ledit revêtement établit un isomorphisme entre un voisinage de $P^{\prime}$ dans $W \times_{V} U$ et un voisinage de $P$ dans $U$. On peut donc se ramener, quitte à restreindre encore $V, U$ et $W$, au cas où $U \rightarrow V$ se factorise par un morphisme fini $\psi: U \rightarrow W$. Par construction de $W$ le corps $\mathcal{H}(\psi(P))$ est séparablement clos dans $\mathcal{H}(P)$. Notons que $X$ étant réduit, $V$ l'est aussi; et comme la dimension de $X$ est inférieure ou égale 
à $n$ et comme $\rho(\mathcal{H}(P) / k)=n$, la dimension de $V$ est exactement $n$. Enfin en restreignant encore $V$ (et par suite $U$ et $W$ ), on peut faire l'hypothèse que $P$ est le seul antécédent de $\varphi(P)$ dans $U$.

La flèche $W \rightarrow V$ est un revêtement fini étale et donc $W$ est également réduit et de dimension $n$. On peut alors appliquer le lemme 1.8 à la flèche $U \rightarrow W$ avec $P($ resp. $\psi(P))$ dans le rôle de $y$ (resp. $x)$. On en déduit l'existence d'un ouvert Zariski $\Omega$ de $W$ contenant $\psi(P)$ tel que $\psi^{-1}(\Omega) \rightarrow \Omega$ soit fini, radiciel et plat. Soit $V^{\prime}$ un voisinage strictement $k$-analytique de $\varphi(P)$ dans $V$ tel que $\left(\varphi_{\mid U}\right)^{-1}\left(V^{\prime}\right)$ soit inclus dans $\psi^{-1}(\Omega)$; on conclut en remplaçant $V$ (resp. $W$, resp. $U$ ) par $V^{\prime}$ (resp. par l'image réciproque de $V^{\prime}$ dans $W$, resp. par l'image réciproque de $V^{\prime}$ dans $U$ ).

\section{Morphismes discrets entre espaces linéaires par morceaux}

2.1. Définition. - Soit $X$ un espace linéaire par morceaux. On dira que $X$ est un pseudo-polytope s'il existe un isomorphisme $\varphi$ entre $X$ et un polytope $Y$ de $\left(\mathbb{R}_{+}^{*}\right)^{m}$ pour un certain $m$. On appellera intérieur d'un pseudo-polytope $X$ l'ensemble des points de $X$ possédant un voisinage homéomorphe à une boule ouverte. Le bord de $X$ est le complémentaire de son intérieur.

2.2. Soit $X$ un pseudo-polytope et $n$ sa dimension. On appellera système de coordonnées sur $X$ tout $n$-uplet $\left(t_{1}, \ldots, t_{n}\right)$ de fonctions linéaires par morceaux sur $X$ qui induit un isomorphisme entre $X$ et un polytope de $\left(\mathbb{R}_{+}^{*}\right)^{n}$. Soit $\left(t_{i}\right)$ un tel système de coordonnées sur $X$. Soit $p$ un entier; une application de $X$ dans $\left(\mathbb{R}_{+}^{*}\right)^{p}$ sera dite affine relativement au système des $t_{i}$ si chacune de ses coordonnées est de la forme $r \prod t_{i}^{a_{i}}$ où $r$ est un élément de $\sqrt{\left|k^{*}\right|}$ et où les $a_{i}$ appartiennent à $\mathbb{Q}$. Une application $\varphi$ de $X$ vers un pseudo-polytope $X^{\prime}$ de dimension $p$ muni d'un système de coordonnées $\left(t_{1}^{\prime}, \ldots, t_{p}^{\prime}\right)$ sera dite affine relativement au système des $t_{i}$ et à celui des $t_{j}^{\prime}$ si pour tout $j$ la composée $t_{j}^{\prime} \circ \varphi$ est affine relativement au système des $t_{i}$.

2.3. Proposition. - Soit $X$ un espace linéaire par morceaux, soit $Y$ un espace topologique et soit $\varphi: Y \rightarrow X$ une application continue à fibres discrètes. Convenons d'une dire qu'une structure linéaire par morceaux sur $Y$ est compatible avec $\varphi$ si elle fait de $\varphi$ une application linéaire par morceaux.

(i) Il existe alors au plus une structure linéaire par morceaux sur $Y$ compatible avec $\varphi$.

(ii) Si une telle structure existe, alors $\varphi$ est G-localement une immersion.

(iii) Si une telle structure existe et si $Z$ est un sous-ensemble de $Y$ muni d'une structure linéaire par morceaux compatible avec $\varphi$, structure qui est nécessairement unique d'après le (i), alors $Z$ est un sous-espace linéaire par morceaux de $Y$.

BULlETIN DE LA SOCIÉTÉ MATHÉMATIQUE DE FRANCE 
Démonstration. - Supposons $Y$ muni d'une structure linéaire par morceaux $\mathcal{S}$ compatible avec $\varphi$. On se propose caractériser $\mathcal{S}$ à l'aide des propriétés intrinsèques de la flèche $Y \rightarrow X$. La démonstration repose sur plusieurs lemmes.

2.3.1. Lemme. - Soit $W$ un pseudo-polytope de $X$. L'espace linéaire par morceaux $\varphi^{-1}(W)$ possède un $G$-recouvrement par une famille $\left(V_{i}\right)$ où $V_{i}$ est pour tout $i$ un pseudo-polytope de $Y$ tel que $\varphi_{\mid V_{i}}$ soit injective, et donc établisse un isomorphisme entre $V_{i}$ et $\varphi\left(V_{i}\right)$.

Démonstration. - Choisissons un système de coordonnées sur $W$. Comme $\varphi$ est linéaire par morceaux, $\varphi^{-1}(W)$ est un sous-espace linéaire par morceaux de $Y$. Il possède donc un $G$-recouvrement par une famille de pseudo-polytopes $\left(V_{i}\right)$ telle que pour tout $i$ l'application $\varphi_{\mid V_{i}}$ soit affine relativement à un système de coordonnées convenable sur $V_{i}$ et à celui choisi sur $W$. Comme $\varphi_{\mid V_{i}}$ est à fibres discrètes, elle est injective.

2.3.2. Lemme. - Soient $W$ un pseudo-polytope de $X$ et $\sigma$ une section continue de $\varphi$ sur $W$. L'application $\sigma$ est linéaire par morceaux.

Démonstration. - D'après le lemme précédent, il existe un $G$-recouvrement de $\varphi^{-1}(W)$ par une famille $\left(V_{i}\right)$ où chaque $V_{i}$ est un pseudo-polytope tel que $\varphi_{\mid V_{i}}$ établisse un isomorphisme entre $V_{i}$ et $\varphi\left(V_{i}\right)$. Comme $\sigma(W)$ est compact, il existe un ensemble fini $I$ d'indices tel que $\sigma(W) \subset \bigcup_{I} V_{i}$. Soit $n$ la dimension de $W$. Notons que pour tout $i$ le pseudo-polytope $V_{i}$ s'injecte de manière affine dans $W$ et est donc de dimension inférieure ou égale à $n$. Soit $J$ l'ensemble des indices $j$ appartenant à $I$ tels que $V_{j}$ soit de dimension $n$ et tel que $\sigma(W)$ rencontre l'intérieur $V_{j}^{\prime}$ de $V_{j}$. Soit $j$ appartenant à $J$. Il existe un point $P$ appartenant à $W$ tel que $\sigma(P)$ appartienne à $V_{j}^{\prime}$. Comme $V_{j}^{\prime}$ est connexe, il en va de même de $\sigma\left(\varphi\left(V_{j}^{\prime}\right)\right)$. Désignons par $U_{j}$ le complémentaire de $V_{j}$ dans $\bigcup_{I} V_{i}$. Comme $\sigma\left(\varphi\left(V_{j}^{\prime}\right)\right)$ ne rencontre pas le bord de $V_{j}$, on peut l'écrire comme la réunion disjointe des deux ouverts $U_{j} \cap \sigma\left(\varphi\left(V_{j}^{\prime}\right)\right)$ et $V_{j}^{\prime} \cap \sigma\left(\varphi\left(V_{j}^{\prime}\right)\right)$. Comme $\sigma\left(\varphi\left(V_{j}^{\prime}\right)\right)$ est connexe et rencontre $V_{j}^{\prime}$, on a $\sigma\left(\varphi\left(V_{j}^{\prime}\right) \subset V_{j}^{\prime}\right.$; comme $\varphi_{\mid V_{j}^{\prime}}$ est injective, l'application $\sigma \circ \varphi_{\mid V_{j}^{\prime}}$ est l'identité. Par continuité (l'espace $V_{j}$ est séparé) $\sigma \circ \varphi_{\mid V_{j}}=\operatorname{Id}_{V_{j}}$. La restriction de $\sigma$ à $\varphi\left(V_{j}\right)$ est donc la réciproque de $\varphi_{\mid V_{j}}$ et est en particulier linéaire par morceaux.

Soit $P$ appartenant à $W$. Comme tout voisinage de $P$ contient un sousensemble homéomorphe à une boule ouverte de dimension $n$, l'image par $\sigma$ de tout voisinage de $P$ rencontre l'intérieur de $V_{j}$ pour un certain $j$ appartenant à $J$ et donc tout voisinage de $P$ rencontre la réunion des $\varphi\left(V_{j}\right)$ où $j$ parcourt $J$. Cette réunion est donc un sous-ensemble compact et dense de $W$, c'est par conséquent $W$ lui-même. Et comme $\sigma_{\mid \varphi\left(V_{j}\right)}$ est d'après ce qui précède linéaire par morceaux pour tout $j$ appartenant à $J$, on en déduit que $\sigma$ est linéaire par morceaux.

TOME $131-2003-\mathrm{N}^{\mathrm{O}} 4$ 
2.3.3. Suite de la démonstration de la proposition 2.3. - Soient $W$ un pseudopolytope de $X$ et $\sigma$ une section continue de $\varphi$ définie sur $W$. D'après ce qui précède, $\sigma$ est linéaire par morceaux et bien sûr propre. Son image $\sigma(W)$ est donc un sous-espace linéaire par morceaux de $Y(c f .[1], \S 1.3)$; sa structure, qui est celle induite par $\mathcal{S}$, peut également être décrite comme la structure linéaire par morceaux déduite via $\sigma$ de celle de $W$.

Considérons la famille $\mathcal{T}$ des $\sigma(W)$ où $W$ parcourt l'ensemble des pseudopolytopes de $X$ et où $\sigma$ parcourt, pour $W$ fixé, l'ensemble des sections continues de $\varphi$ sur $W$; on munit chacun des $\sigma(W)$ de la structure de polyèdre déduite via $\sigma$ de celle de $W$. On va montrer que $\mathcal{T}$ constitue un atlas linéaire par morceaux sur $Y$ et que la structure qu'il définit est précisément la structure $\mathcal{S}$ fixée sur $Y$. Ceci prouvera bien que cette dernière peut se définir uniquement en termes des propriétés intrinsèques de l'application continue $\varphi$, et achèvera la démonstration de (i) et de (ii), le fait que $\varphi$ soit $G$-localement une immersion découlant immédiatement de la définition de $\mathcal{T}$.

Notons déjà que d'après ce qui précède chaque élément de $\mathcal{T}$ est un polyèdre pour $\mathcal{S}$; il suffit donc de montrer que tout point de $Y$ possède un voisinage qui est réunion finie d'éléments de $\mathcal{T}$. Soit $P$ appartenant à $Y$. Il existe un voisinage de $\varphi(P)$ dans $X$ qui est réunion d'un nombre fini de polyèdres, et même de pseudo-polytopes; il suffit donc de montrer que pour tout pseudo-polytope $\mathcal{P}$ de $X$, l'ensemble $\varphi^{-1}(\mathcal{P})$ possède un $G$-recouvrement par des éléments de $\mathcal{T}$. D'après le lemme 2.3.1, le sous-espace linéaire par morceaux $\varphi^{-1}(\mathcal{P})$ de $Y$ possède un $G$-recouvrement par une famille $\left(V_{i}\right)$ de pseudo-polytopes telle que $\varphi_{\mid V_{i}}$ établisse pour tout $i$ un isomorphisme entre $V_{i}$ et $\varphi\left(V_{i}\right)$. Fixons $i$. Si l'on note $\sigma$ la réciproque de $\varphi_{\mid V_{i}}: V_{i} \rightarrow \varphi\left(V_{i}\right)$ on a alors $V_{i}=\sigma\left(\varphi\left(V_{i}\right)\right)$, ce qui montre que $V_{i}$ appartient à $\mathcal{T}$. La démonstration de (i) et (ii) est achevée.

2.3.4. Montrons maintenant (iii). On suppose donc $Y$ munie d'une structure linéaire par morceaux compatible avec $\varphi$. Soit $Z$ un sous-ensemble de $Y$ muni d'une structure linéaire par morceaux compatible avec $\varphi$. L'application continue $\varphi_{\mid Z}$ est bien sûr à fibres discrètes. Les résultats établis au $§ 2.3 .3$ s'appliquent à $Z$; la structure linéaire par morceaux de ce dernier est donc donnée par l'atlas formé des $\sigma(W)$ où $W$ parcourt l'ensemble des pseudo-polytopes de $X$ et où $\sigma$ parcourt, $W$ étant fixé, l'ensemble des sections de $\varphi$ définies sur $W$ et à valeurs dans $Z$. En particulier tout point de $Z$ possède un voisinage qui est réunion finie de tels $\sigma(W)$. Or chacun de ces $\sigma(W)$ est un polyèdre pour la structure linéaire par morceaux de $Y$ (toujours d'après le $\S 2.3 .3$ ); en conséquence $Z$ est bien un sous-espace linéaire par morceaux de $Y$. La démonstration est terminée.

2.4. Corollaire. - Soit $X$ un espace topologique séparé et localement compact et soit $\left(X_{i}\right)$ une famille de sous-ensembles de $X$ tel que tout point de $X$ 
possède un voisinage qui soit réunion d'un nombre fini de $X_{i}$. Soit $\varphi$ une application continue à fibres discrètes de $X$ vers un espace linéaire par morceaux $Y$. Supposons que pour tout couple $(i, j)$ il existe une structure linéaire par morceaux $\mathcal{S}_{(i, j)}$ sur $X_{i} \cap X_{j}$ compatible avec $\varphi$. Alors il existe une unique structure linéaire par morceaux sur $X$ compatible avec $\varphi$ et $\varphi$ est $G$-localement une immersion.

Démonstration. - Comme les fibres de $\varphi$ sont discrètes on déduit de la proposition 2.3 ci-dessus que pour tout couple $(i, j)$ les structures $\mathcal{S}_{i, j}$ et $\mathcal{S}_{j, i}$ cö̈ncident et font de $X_{i} \cap X_{j}$ un sous-espace linéaire par morceaux de $X_{i}$ (muni de $\mathcal{S}_{(i, i)}$ ) aussi bien que de $X_{j}$ (muni de $\mathcal{S}_{(j, j)}$ ). Il est alors immédiat que la réunion des atlas linéaires par morceaux de chacune des structures $S_{(i, i)}$ constitue un atlas linéaire par morceaux sur $X$ qui définit une structure compatible avec $\varphi$. L'unicité de la structure linéaire par morceaux sur $X$ et le fait que $\varphi$ soit $G$-localement une immersion proviennent de la proposition 2.3.

2.5. Corollaire. - Soient $X, Y$ et $Z$ trois espaces linéaires par morceaux, soit $\varphi: Y \rightarrow Z$ une application linéaire par morceaux à fibres discrètes et soit $\psi: X \rightarrow Y$ une application continue à fibres discrètes telle que $\varphi \circ \psi$ soit linéaire par morceaux. Alors $\psi$ est linéaire par morceaux.

Démonstration. - L'application $\varphi \circ \psi$ étant linéaire par morceaux et à fibres discrètes elle est $G$-localement une immersion d'après la proposition 2.3. Il existe donc un $G$-recouvrement de $X$ par une famille $\left(\mathcal{P}_{i}\right)$ de polyèdres tels que $\varphi \circ \psi_{\mid \mathcal{P}_{i}}$ soit injective. Pour tout $i$ l'application $\varphi \circ \psi$ établit par conséquent un isomorphisme linéaire par morceaux entre $\mathcal{P}_{i}$ et $\varphi \circ \psi\left(\mathcal{P}_{i}\right)$, et $\psi$ (resp. $\varphi$ ) induit un homéomorphisme entre $\mathcal{P}_{i}$ et $\psi\left(\mathcal{P}_{i}\right)$ (resp. entre $\psi\left(\mathcal{P}_{i}\right)$ et $\left.\varphi \circ \psi\left(\mathcal{P}_{i}\right)\right)$. Dès lors $\psi\left(\mathcal{P}_{i}\right)$ est un sous-ensemble de $Y$ pouvant être muni d'une structure linéaire par morceaux compatible avec $\varphi$, à savoir la structure déduite via $\varphi$ de celle de $\varphi \circ \psi\left(\mathcal{P}_{i}\right)$. La proposition 2.3 implique alors que $\psi\left(\mathcal{P}_{i}\right)$ est un sous-espace linéaire par morceaux de $Y$. De plus $\psi_{\mid \mathcal{P}_{i}}$ est égale à $\left(\varphi_{\mid \psi\left(\mathcal{P}_{i}\right)}\right)^{-1} \circ\left(\varphi \circ \psi_{\mid \mathcal{P}_{i}}\right)$ et est donc linéaire par morceaux. Comme ceci est vrai quelque soit $i$ et comme la famille des $\mathcal{P}_{i}$ constitue un $G$-recouvrement de $X$ l'application $\psi$ est linéaire par morceaux.

\section{Le théorème principal et sa preuve}

Le théorème principal est le suivant :

3.1. ThÉORÈme. - Soit $k$ un corps valué complet. Soient $n$ un entier et $\mathfrak{X}$ un $k^{0}$-schéma formel pluristable non dégénéré purement de dimension $n$. Soit $Z$ un espace strictement $k$-analytique topologiquement séparé de dimension inférieure ou égale à $n$ et $\varphi$ un morphisme de $Z$ vers $\mathfrak{X}_{\eta}$. Posons

$$
\Delta=\varphi^{-1}(S(\mathfrak{X})) .
$$

TOME $131-2003-\mathrm{N}^{\mathrm{O}} 4$ 
Il existe alors une unique structure linéaire par morceaux sur $\Delta$ telle que

$$
\varphi_{\mid \Delta}: \Delta \longrightarrow S(\mathfrak{X})
$$

soit linéaire par morceaux et $\varphi_{\mid \Delta}$ est $G$-localement une immersion.

Démonstration. - Elle comprend quatre étapes.

Première étape : on se réduit au cas où $\mathfrak{X}$ est strictement pluristable non dégénéré.

3.2. Remarque préliminaire. - En vertu des lemmes 1.5 et 1.9, la fibre de $\varphi$ en tout point de $S(\mathfrak{X})$ est discrète.

3.3. Supposons que l'on sache montrer le théorème lorsque $\mathfrak{X}$ est strictement pluristable et non dégénéré; on va expliquer comment l'en déduire dans le cas général. Choisissons un morphisme étale surjectif et quasi-compact $\mathfrak{Y} \rightarrow \mathfrak{X}$ où $\mathfrak{Y}$ est strictement pluristable non dégénéré. Posons $Z^{\prime}=Z \times_{\mathfrak{X}_{\eta}} \mathfrak{Y}_{\eta}$.

3.4. Considérons le diagramme commutatif suivant dont les flèches verticales sont surjectives (cela se voit en utilisant le lemme 2.2 de [4]) :

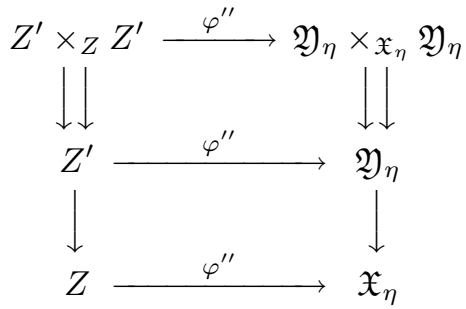

3.5. On rappelle que $\Delta=\varphi^{-1}(S(\mathfrak{X}))$. On note de même $\Delta^{\prime}$ (resp. $\Delta^{\prime \prime}$ ) l'image réciproque de $S(\mathfrak{Y})($ resp. de $S(\mathfrak{Y} \times \mathfrak{X} \mathfrak{Y}))$ par $\varphi^{\prime}\left(\right.$ resp. $\left.\varphi^{\prime \prime}\right)$. Comme $S(\mathfrak{Y} \times \mathfrak{X} \mathfrak{Y})$ (resp. $S(\mathfrak{Y})$ ) est l'image réciproque de $S(\mathfrak{Y})$ (resp. de $S(\mathfrak{X})$ ) par chacune des deux projections $\mathfrak{Y}_{\eta} \times_{\mathfrak{X}_{\eta}} \mathfrak{Y}_{\eta} \rightarrow \mathfrak{Y}_{\eta}$ (resp. par la flèche $\mathfrak{Y}_{\eta} \rightarrow \mathfrak{X}_{\eta}$ ) on en déduit que $\Delta^{\prime \prime}$ (resp. $\Delta^{\prime}$ ) est l'image réciproque de $\Delta^{\prime}$ (resp. de $\Delta$ ) par chacune des deux flèches $Z^{\prime} \times{ }_{Z} Z^{\prime} \rightarrow Z^{\prime}$ (resp. par la flèche $Z^{\prime} \rightarrow Z$ ). Comme le produit tensoriel complété de deux corps valués complet au-dessus d'un troisième est non nul on en déduit que $\Delta$ est ensemblistement le conoyau du diagramme

$$
\Delta^{\prime \prime} \Longrightarrow \Delta^{\prime}
$$

Comme $Z^{\prime} \rightarrow Z$ est un recouvrement quasi-étale et comme $\Delta$ est un fermé de $Z$, on déduit du lemme 5.11 de [5] que $\Delta$ est topologiquement le conoyau du diagramme $\Delta^{\prime \prime} \Longrightarrow \Delta^{\prime}$.

3.6. On s'est placé sous l'hypothèse où l'on savait démontrer le théorème 3.1 dans le cas strictement pluristable. Les schémas formels $\mathfrak{Y}$ et $\mathfrak{Y} \times \mathfrak{X} \mathfrak{Y}$ sont strictement pluristables non dégénérés et purement de dimension $n$ et les espaces strictement $k$-analytiques $Z^{\prime}$ et $Z^{\prime} \times{ }_{Z} Z^{\prime}$ sont de dimension au plus $n$. On peut en conséquence munir $\Delta^{\prime}$ (resp. $\left.\Delta^{\prime \prime}\right)$ d'une structure linéaire par morceaux 
compatible avec $\varphi^{\prime}{ }_{\Delta^{\prime}}$ (resp. avec $\left.\varphi^{\prime \prime}{ }_{\mid \Delta^{\prime \prime}}\right)$; de plus dans ce cas $\varphi^{\prime \prime}{ }_{\mid \Delta^{\prime \prime}}$ et $\left.\varphi^{\prime}\right|_{\Delta^{\prime}}$ sont $G$-localement des immersions, et il en va de même de $S(\mathfrak{Y} \times \mathfrak{X} \mathfrak{Y}) \rightarrow S(\mathfrak{Y})$ (puisque c'est un morphisme à fibres finies). Soient $p$ l'une des deux projections $\mathfrak{Y}_{\eta} \times \mathfrak{X}_{\eta} \mathfrak{Y}_{\eta} \rightarrow \mathfrak{Y}_{\eta}$ et $q$ la flèche correspondante de $Z^{\prime} \times{ }_{Z} Z^{\prime}$ vers $Z^{\prime}$; on a donc $\varphi^{\prime} \circ q=p \circ \varphi^{\prime \prime}$. Dès lors $\varphi^{\prime} \circ q$ est linéaire par morceaux. Or $\varphi^{\prime}$ est linéaire par morceaux et à fibres discrètes au-dessus de $S(\mathfrak{Y})$ et $q$ est également à fibres discrètes puisque quasi-étale. Le corollaire 2.5 assure alors que $q_{\mid \Delta^{\prime \prime}}: \Delta^{\prime \prime} \rightarrow \Delta^{\prime}$ est linéaire par morceaux.

On sait que l'espace linéaire par morceaux $S(\mathfrak{X})$ est le conoyau du diagramme

$$
S(\mathfrak{Y} \times \mathfrak{X} \mathfrak{Y}) \Longrightarrow S(\mathfrak{Y}) \text {. }
$$

On a vu par ailleurs que $\Delta$ est topologiquement le conoyau $\Delta^{\prime \prime} \Longrightarrow \Delta^{\prime}$. Or les deux flèches de $\Delta^{\prime \prime}$ vers $\Delta^{\prime}$ sont linéaires par morceaux d'après ce qui précède; on peut donc munir $\Delta$ d'une structure linéaire par morceaux compatible avec $\varphi$, à savoir celle de conoyau dans la catégorie des espaces linéaires par morceaux (cf. [1], prop. 3.6.1) du diagramme $\Delta^{\prime \prime} \Longrightarrow \Delta^{\prime}$.

On s'est donc bien ramené au cas où $\mathfrak{X}$ est strictement pluristable non dégénéré.

Deuxième étape : on remplace $\mathfrak{X}_{\eta}$ par $\left(\mathbb{G}_{m, k}^{\text {an }}\right)^{n}$ et $S(\mathfrak{X})$ par $S_{k}^{n}$.

3.7. On suppose donc à partir de maintenant que $\mathfrak{X}$ est strictement pluristable non dégénéré. Berkovich (voir [1], §5.5), a démontré que $\mathfrak{X}$ possédait un recouvrement ouvert par une famille $\left(\mathfrak{X}_{i}\right)$ de schémas formels telle que chacun des $\mathfrak{X}_{i}$ admette une bonne fibration. Pour tout $i$ on désigne par $Z_{i}$ l'image réciproque de $\mathfrak{X}_{i, \eta}$ par $\varphi$. Si l'on sait munir pour tout $i$ et pour tout domaine strictement $k$-analytique $T$ de $Z_{i}$ l'ensemble $T \cap \Delta$ d'une structure linéaire par morceaux compatible avec $\varphi$, alors le corollaire 2.4 appliqué à la famille des $\Delta \cap Z_{i}$ permet de conclure à la validité du théorème. On se ramène ainsi au cas où $\mathfrak{X}$ lui-même admet une bonne fibration

$$
\mathfrak{X}=\mathfrak{X}_{\ell} \rightarrow \mathfrak{Z}_{\ell} \rightarrow \mathfrak{X}_{\ell-1} \rightarrow \mathfrak{Z}_{\ell-1} \rightarrow \mathfrak{X}_{\ell-2} \rightarrow \cdots \rightarrow \mathfrak{Z}_{0}=\operatorname{Spf} k^{0}
$$

On reprend les notations $\mathbf{n}_{j}, \mathbf{a}_{j}, m_{j}$ introduites au 0.9 lors de la définition d'une bonne fibration.

3.8. Pour tout $j$ compris entre 0 et $\ell$ l'image de $S(\mathfrak{X})$ dans $\mathfrak{X}_{j, \eta}$ (resp. dans $\mathfrak{Z}_{j, \eta}$ ) appartient à $S\left(\mathfrak{X}_{j}\right)$ (resp. à $S\left(\mathfrak{Z}_{j}\right)$ ). Fixons $j$ entre 1 et $\ell$. On a

$$
\mathfrak{Z}_{j} \simeq \mathfrak{X}_{j-1}\left(\mathbf{n}_{j}, \mathbf{a}_{j}, m_{j}\right)
$$

c'est-à-dire encore

$$
\mathfrak{Z}_{j} \simeq \mathfrak{X}_{j-1}\left(\mathbf{n}_{j}, \mathbf{a}_{j}\right) \times_{k^{0}} \mathcal{G}_{m}^{m_{j}}
$$

où l'on a désigné par $\mathcal{G}_{m}$ le schéma formel

$$
\operatorname{Spf}(k\{T, S\} /(T S-1)) .
$$

TOME $131-2003-\mathrm{N}^{\mathrm{O}} 4$ 
Tout point de $S\left(\mathfrak{Z}_{j}\right)$ s'envoie par la seconde projection sur l'unique point $P_{j}$ du bord de Shilov de $\mathcal{G}_{m, \eta}^{m_{j}}$. Supposons que l'ensemble des entiers $j$ compris entre 1 et $\ell$ tels que $m_{j}$ soit non nul est non vide et soit $j_{0}$ son plus petit élément. Remplaçons :

- $k \operatorname{par} \mathcal{H}\left(P_{j_{0}}\right)$ (notons que comme $\left|\mathcal{H}\left(P_{j_{0}}\right)^{*}\right|=\left|k^{*}\right|$, les notions de structure

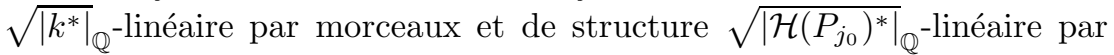
morceaux coïncident);

- chacun des schémas formels $\mathfrak{X}_{j}$ et $\mathfrak{Z}_{j}$ pour $j<j_{0}$ par son produit fibré avec $\mathcal{H}\left(P_{j_{0}}\right)^{0}$ au-dessus de $k^{0}$;

- chacun des schémas formels $\mathfrak{X}_{j}$ et $\mathfrak{Z}_{j}$ pour $j \geq j_{0}$ par son produit fibré avec $\mathfrak{X}_{j_{0}-1}\left(\mathbf{n}_{j_{0}}, \mathbf{a}_{j_{0}}\right) \times_{k^{0}} \mathcal{H}\left(P_{j_{0}}\right)^{0}$ au-dessus de $\mathfrak{Z}_{j_{0}} \simeq \mathfrak{X}_{j_{0}-1}\left(\mathbf{n}_{j_{0}}, \mathbf{a}_{j_{0}}\right) \times_{k^{0}} \mathcal{G}_{m}^{m_{j_{0}}} ;$

- l'espace strictement $k$-analytique $Z$ par la fibre en $P_{j_{0}}$ de la flèche composée

$$
Z \longrightarrow \mathfrak{X}_{\eta} \longrightarrow \mathfrak{Z}_{j_{0}, \eta} \simeq \mathfrak{X}_{j_{0}-1}\left(\mathbf{n}_{j_{0}}, \mathbf{a}_{j_{0}}\right)_{\eta} \times_{k} \mathcal{G}_{m, \eta}^{m_{j_{0}}} \longrightarrow \mathcal{G}_{m, \eta}^{m_{j_{0}}}
$$

On se ramène ainsi au cas où $m_{j}$ est nul pour tout entier $j$ inférieur ou égal à $j_{0}$. En recommençant l'opération autant de fois qu'il est nécessaire, on se ramène finalement à considérer la situation où tous les $m_{j}$ sont nuls. Sous cette hypothèse on dispose d'une description simple de $S(\mathfrak{X})$, description fondée sur certains calculs explicites de Berkovich ( $c f$. [5], étape 3 de la preuve du théorème 5.2) ainsi que sur la remarque 0.11.

3.9. Description de $S(\mathfrak{X})$. - On suppose donc que $\mathfrak{X}$ peut être munie d'une fibration de la forme décrite au 3.7 pour laquelle les $m_{j}$ sont tous nuls. Le squelette $S(\mathfrak{X})$ est alors un polyèdre de dimension exactement $n$. De plus il existe un $n$-uplet $\mathbf{g}=\left(g_{1}, \ldots, g_{n}\right)$ de fonctions appartenant à $\mathcal{O}(\mathfrak{X})$ telles que :

- la famille des $\left|g_{i}\right|_{\mid S(\mathfrak{X})}$ constitue un système de coordonnées sur $S(\mathfrak{X})$;

- pour tout point $P$ de $\mathfrak{X}_{\eta}$ les propositions suivantes sont équivalentes :

(i) $P$ appartient à $S(\mathfrak{X})$;

(ii) pour tout polynôme $\sum a_{I} \mathbf{T}^{I}$ en $n$ variables à coefficients dans $k$, on a l'égalité

$$
\left|\sum a_{I} \mathbf{g}^{I}(P)\right|=\max _{I}\left|a_{I}\right| \cdot|\mathbf{g}(P)|^{I} .
$$

3.10. Sur $S(\mathfrak{X})$, les $g_{i}$ sont inversibles; on peut donc remplacer $Z$ par le lieu d'inversibilité des $g_{i}$ sans changer $\varphi^{-1}(S(\mathfrak{X}))$. Le $n$-uplet $\left(g_{1}, \ldots, g_{n}\right)$ définit alors un morphisme de $Z$ vers $\left(\mathbb{G}_{m, k}^{\text {an }}\right)^{n}$ et $\Delta$ est l'image réciproque par cette flèche du fermé $S_{k}^{n}$ qui a été défini au $\S 0.10$; rappelons que $S_{k}^{n}$ est naturellement muni d'un homéomorphisme avec $\left(\mathbb{R}_{+}^{*}\right)^{n}$ via lequel on en fait un espace linéaire par morceaux.

L'application $\Delta \rightarrow S_{k}^{n}$ ainsi induite par $Z \mapsto\left(\mathbb{G}_{m, k}^{\text {an }}\right)^{n}$ se factorise par $S(\mathfrak{X})$ et $S(\mathfrak{X}) \rightarrow S_{k}^{n}$ est une immersion d'espaces linéaires par morceaux. 
Il suffit par conséquent de montrer que $\Delta$ peut être muni d'une structure linéaire par morceaux compatible avec $\Delta \rightarrow S_{k}^{n}$.

3.11. Récapitulation. - On se donne un espace strictement $k$-analytique $Z$ topologiquement séparé et de dimension inférieure ou égale à $n$. Soit $\varphi$ un morphisme $Z \rightarrow\left(\mathbb{G}_{m, k}^{\text {an }}\right)^{n}$; notons $\Delta$ le fermé $\varphi^{-1}\left(S_{k}^{n}\right)$ de $Z$. On cherche à montrer que $\Delta$ peut être muni d'une structure linéaire par morceaux compatible avec $\varphi$. Pour le problème auquel on s'intéresse seul importe l'espace topologique sous-jacent $\grave{a} Z$; on peut donc supposer que $Z$ est réduit.

Troisième étape : on se ramène au cas où $\varphi$ est un morphisme fini étale d'un espace algébroïde sur un domaine de $\left(\mathbb{G}_{m, k}^{\mathrm{an}}\right)^{n}$. Le contexte est celui du $\S 3.11$ ci-dessus.

3.12. Soit $P$ un point de $\Delta$ et soit $Y$ un domaine strictement $k$-affinoïde de $Z$ contenant $P$. La dimension de $Z$ est inférieure ou égale à $n$; il en va donc de même de celle de $Y$. Par ailleurs $\varphi(P)$ appartient à $S_{k}^{n}$ et donc

$$
\rho(\mathcal{H}(\varphi(P)) / k)=n
$$

d'après l'exemple 1.4. On en déduit que $\rho(\mathcal{H}(P) / k) \geq n$ puis finalement, en utilisant le lemme 1.6, que $\rho(\mathcal{H}(P) / k)=n$ et que la dimension de $Y$ est exactement $n$. D'après la version analytique du lemme de normalisation de Nœther ( $c f$. [6], cor. 2, p. 228), il existe un morphisme fini $\psi$ de $Y$ vers $\mathbb{D}^{n}$, où $\mathbb{D}$ désigne le disque unité de dimension 1 . La finitude de $\psi$ entrâne l'égalité $\rho(\mathcal{H}(\psi(P)) / k)=n$. Le lemme 1.9 s'applique alors et assure l'existence d'un voisinage strictement $k$-affinoïde $U$ de $P$ dans $Y$ et d'un voisinage strictement $k$-affinoïde $V$ de $\psi(P)$ dans $\mathbb{D}^{n}$ tels que $\psi(U) \subset V$ et tels que la flèche $U \rightarrow V$ ainsi induite se factorise sous la forme $U \rightarrow W \rightarrow V$ où $U \rightarrow W$ et $W \rightarrow V$ sont deux revêtements finis et plats respectivement radiciel et étale. On peut toujours supposer que $U, V$ et $W$ sont irréductibles (et même intègres puisque $Z$ est réduit) et, en utilisant le caractère algébroïde de $\mathbb{D}^{n}$ et la remarque 0.5 , que $W$ est algébroïde.

3.13. Le corollaire 2.4 permet, de manière analogue à ce qui a été fait au 3.7 , de raisonner $G$-localement sur $Z$ et, en se fondant sur les remarques faites au 3.12 ci-dessus, de modifier la question étudiée.

3.14. Nouvelle version du problème à traiter. - Soit $U$ un espace strictement $k$-affinoïde intègre muni d'une flèche vers un domaine strictement $k$-affinoïde $V$ de $\mathbb{D}^{n}$ qui se factorise sous la forme $U \rightarrow W \rightarrow V$ où $W$ est strictement $k$-affinoïde et algébroïde et où $U \rightarrow W$ et $W \rightarrow V$ sont deux revêtements finis et plats respectivement radiciel et étale. Soit $\varphi$ un morphisme de $U$ vers $\left(\mathbb{G}_{m, k}^{\text {an }}\right)^{n}$ et $\Delta$ l'image réciproque de $S_{k}^{n}$ par $\varphi$. On souhaite montrer que pour tout domaine strictement $k$-analytique $T$ de $U$, l'intersection $T \cap \Delta$ peut être munie d'une structure linéaire par morceaux compatible avec $\varphi$. 
3.15. On travaille désormais avec les hypothèses et notations du $\S 3.14$ cidessus. Notons $\left(g_{1}, \ldots, g_{n}\right)$ les $n$ fonctions inversibles sur $U$ qui définissent $\varphi$ et $\mathbf{g}$ le $n$-uplet correspondant. D'après le $\S 0.14$, il existe alors un entier $r$ strictement positif, puissance de la caractéristique de $k$, tel que pour tout $f$ appartenant à $\mathcal{O}(U)$ la fonction $f^{r}$ provienne de $\mathcal{O}(W)$. Notons $\varphi^{r}$ le morphisme $U \rightarrow\left(\mathbb{G}_{m, k}^{\text {an }}\right)^{n}$ défini par le $n$-uplet $\left(g_{1}^{r}, \ldots, g_{n}^{r}\right)$. Comme $r$ est une puissance de la caractéristique de $k$ les assertions suivantes sont équivalentes pour tout point $P$ de $U$ :

(i) $\varphi(P)$ appartient à $S_{k}^{n}$,

(ii) $\varphi^{r}(P)$ appartient à $S_{k}^{n}$.

Pour tout $i$, la fonction $g_{i}^{r}$ provient de $\mathcal{O}(W)$. En conséquence $\varphi^{r}$ est la composée de $U \rightarrow W$ et d'un certain morphisme $\psi: W \rightarrow\left(\mathbb{G}_{m, k}^{\text {an }}\right)^{n}$; en vertu de l'équivalence mentionnée ci-dessus la flèche $U \rightarrow W$, qui induit un homéomorphisme entre les espaces topologiques sous-jacents, identifie $\Delta$ et $\psi^{-1}\left(S_{k}^{n}\right)$. D'autre part comme $T$ est un domaine strictement $k$-analytique de $U$, il possède un $G$-recouvrement par des domaines strictement $k$-affinö̈des rationnels (c'est le théorème de Gerritzen-Grauert, $c f$. [6], §7.3.5, cor. 3) et donc définis par des inégalités entre fonctions de $\mathcal{O}(U)$. On peut tout aussi bien remplacer chacune d'elles par sa $r$-ième puissance et donc supposer qu'elle provient de $\mathcal{O}(W)$. Ceci montre que $U \rightarrow W$ identifie $T$ à un domaine strictement $k$-analytique de $W$.

3.16. On peut donc remplacer $U$ par $W, \varphi$ par $\psi$ et $T$ par son image dans $W$. On se ramène ainsi au cas où $U$ est algébrö̈de et muni d'une flèche $\varphi$ vers $\left(\mathbb{G}_{m, k}^{\text {an }}\right)^{n}$. On conserve les notations $\Delta$ et $T$ du $\S 3.14$.

3.17. Le corollaire 2.4 permet une fois encore de raisonner $G$-localement sur $U$ et donc de faire l'hypothèse suivante : il existe une $k$-variété algébrique $\mathcal{X}$, un domaine strictement $k$-affinö̈de $X$ de $\mathcal{X}^{\text {an }}$ et un isomorphisme entre $U$ et un domaine strictement $k$-affinoïde rationnel de $X$ inclus dans $\operatorname{Int} X / \mathcal{X}^{\text {an }}$.

3.18. On note toujours $\left(g_{1}, \ldots, g_{n}\right)$ le $n$-uplet qui définit $\varphi$. Soit $\varepsilon$ un réel strictement positif tel que pour tout $i$ et pour tout point $P$ de $U$ l'on ait $\left|g_{i}(P)\right|>\varepsilon$ (l'existence de $\varepsilon$ est assurée par la compacité de $U$ ). Comme $U$ est un domaine rationnel de $X$, il existe un ouvert $\Omega$ de $X$ contenant $U$ et que l'on peut toujours supposer inclus dans Int $X / \mathcal{X}^{\text {an }}$, et $n$ fonctions $\gamma_{1}, \ldots, \gamma_{n}$ inversibles sur $\Omega$ telles que pour tout $i$ et tout point $P$ de $U$ l'on ait $\left|\gamma_{i}(P)-g_{i}(P)\right|<\varepsilon$. Dès lors si l'on désigne par $\psi$ la flèche $\Omega \rightarrow\left(\mathbb{G}_{m, k}^{\text {an }}\right)^{n}$ induite par les $\gamma_{i}$, alors $\psi_{\mid U}^{-1}\left(S_{k}^{n}\right)=\Delta$. Or $\Omega$ est par construction un ouvert de $\mathcal{X}^{\text {an }}$ et est donc sans bord. En remplaçant $U$ par $\Omega$ et $\varphi$ par $\psi$ on se retrouve dans la situation décrite au $\S 3.16$ avec $U$ de surcroît supposé sans bord.

3.19. Soit $P$ appartenant à $\Delta$. Comme $\varphi(P)$ appartient à $S_{k}^{n}$, on a

$$
\rho(\mathcal{H}(\varphi(P)) / k)=n
$$


d'après l'exemple 1.4. D'autre part comme $U$ est sans bord, $P$ appartient à Int $U /\left(\mathbb{G}_{m, k}^{\text {an }}\right)^{n}$. Notons enfin que tout voisinage de $\varphi(P)$ dans $\left(\mathbb{G}_{m, k}^{\text {an }}\right)^{n}$ contient un pavé de dimension $n$ inclus dans $S_{k}^{n}$. Le lemme 1.9 et le corollaire 2.4 permettent alors de supposer que $U \rightarrow\left(\mathbb{G}_{m, k}^{\text {an }}\right)^{n}$ se factorise par un domaine strictement $k$-affinoïde $V$ de $\left(\mathbb{G}_{m, k}^{\text {an }}\right)^{n}$ contenant un pavé de dimension $n$ de $S_{k}^{n}$ et que la flèche $U \rightarrow V$ admet une décomposition $U \rightarrow W \rightarrow V$ où $U \rightarrow W$ et $W \rightarrow V$ sont deux revêtements finis et plats respectivement radiciel et étale; on peut supposer que $V, W$ et $U$ sont intègres et, grâce à la remarque 0.5 , que $W$ est algébroïde. Enfin puisque $V$ contient un pavé de dimension $n$ inclus dans $S_{k}^{n}$, on peut faire l'hypothèse, en remplaçant $V$ par son intersection avec une polycouronne convenable, que $V \cap S_{k}^{n}$ est un pavé de dimension $n$.

Le raisonnement tenu au $\S 3.15$ peut s'appliquer ici et permet, en remplaçant $U$ par $W$ et $T$ par son image dans $W$, de ramener le problème étudié à celui décrit ci-dessous.

3.20. Problème final. - On se donne un espace strictement $k$-affinoïde et algébroïde intègre $U$ muni d'un morphisme étale $\varphi$ vers un domaine strictement $k$-affinö̈de $V$ de $\left(\mathbb{G}_{m, k}^{\text {an }}\right)^{n}$ qui intersecte $S_{k}^{n}$ selon un pavé $\mathbb{V}$ de dimension $n$. L'image réciproque de $\mathbb{V}$ par $\varphi$ est notée $\Delta$. Soit $T$ un domaine strictement $k$ analytique de $U$. On souhaite montrer que $T \cap \Delta$ peut être muni d'une structure linéaire par morceaux compatible avec $\varphi$. On note $g_{1}, \ldots, g_{n}$ les $n$ fonctions qui définissent $\varphi$ et $\mathbf{g}$ le $n$-uplet correspondant.

Dernière étape de la démonstration : on travaille sous les hypothèses et notations du §3.20 ci-dessus.

3.21. Comme $U$ est algébroïde, il existe une $k$-variété algébrique $\mathcal{X}$ telle que $U$ s'identifie à un domaine strictement $k$-affinoïde de $\mathcal{X}^{\text {an }}$. Berkovich a montré (voir [5], lemme 9.4) que l'on pouvait construire par éclatements successifs un $k^{0}$-schéma $\mathcal{Y}$ intègre, propre et plat possédant la propriété suivante : il existe un ouvert $\mathcal{U}$ de $\mathcal{Y}_{s}$ et une immersion ouverte de $\mathcal{X}$ dans $\mathcal{Y}_{\eta}$ qui identifie $U$ avec le domaine analytique $\pi^{-1}(\mathcal{U})$ de $\mathcal{Y}_{\eta}^{\text {an }} \simeq \widehat{\mathcal{Y}}_{\eta}$.

Berkovich démontre ensuite ([5], étape 3 de la preuve du th. 9.1), en utilisant la théorie des altérations de De Jong, le résultat suivant :

3.22. Proposition (Berkovich, d'après De Jong). - Il existe

(i) une extension $L / k$ finie normale;

(ii) un $L^{0}$-schéma propre, intègre, et pluristable non dégénéré $\mathcal{Y}^{\prime}$, dont la fibre générique est lisse;

(iii) un groupe fini $\mathbf{G}$ agissant sur $\mathcal{Y}^{\prime}$ au-dessus de $k^{0}$;

(iv) un morphisme dominant $\mathbf{G}$-équivariant $\psi: \mathcal{Y}^{\prime} \rightarrow \mathcal{Y}$ (avec action triviale de $\mathbf{G}$ sur $\mathcal{Y}$ ) telle que la flèche $\mathcal{Y}_{\eta}^{\prime} \rightarrow \mathcal{Y}_{\eta}$ induite par $\psi$ soit propre et génériquement finie, et telle que $\kappa\left(\mathcal{Y}_{\eta}^{\prime}\right)^{\mathbf{G}}$ soit une extension purement inséparable de $\kappa\left(\mathcal{Y}_{\eta}\right)$. 
3.23. Les morphismes induits par $\psi$ aux niveaux spécial, générique, analytique, etc. seront encore notés $\psi$. La propriété (iv) implique l'existence d'un ouvert Zariski non vide $\mathcal{V}$ de $\mathcal{Y}_{\eta}$ tel que $\psi^{-1}(\mathcal{V}) \rightarrow \mathcal{V}$ se factorise sous la forme $\psi^{-1}(\mathcal{V}) \rightarrow \mathcal{V}_{0} \rightarrow \mathcal{V}$ où $\psi^{-1}(\mathcal{V}) \rightarrow \mathcal{V}_{0}$ est un revêtement galoisien de groupe $\mathbf{G}$, et où $\mathcal{V}_{0} \rightarrow \mathcal{V}$ est un revêtement fini, radiciel et plat. On pose

$$
U^{\prime}=\psi^{-1}(U), \quad \Delta^{\prime}=\psi^{-1}(\Delta) \quad \text { et } \quad T^{\prime}=\psi^{-1}(T) .
$$

Soit $\mathcal{U}^{\prime}$ l'image réciproque de $\mathcal{U}$ par $\psi$. On a alors $U^{\prime}=\pi^{-1}\left(\mathcal{U}^{\prime}\right)$. Soit $\mathfrak{U}^{\prime}$ le sous-schéma formel ouvert de $\widehat{\mathcal{Y}}^{\prime}$ dont l'espace topologique sous-jacent est $\mathcal{U}^{\prime}$; notons que $\mathfrak{U}_{\eta}^{\prime} \simeq U^{\prime}(c f$. [4], fin du $\S 1)$. On notera encore $g_{1}, \ldots, g_{n}$ les images de ces fonctions dans $\mathcal{O}\left(U^{\prime}\right)$ et $\mathbf{g}$ le $n$-uplet correspondant.

3.24. Lemme. - Le sous-ensemble $\Delta^{\prime}$ de $U^{\prime} \simeq \mathfrak{U}_{\eta}^{\prime}$ est inclus dans $S\left(\mathfrak{U}^{\prime}\right)$.

Démonstration. - Notons que comme $\mathfrak{U}^{\prime}$ est pluristable non dégénéré audessus de $L^{0}$ la notation $S\left(\mathfrak{U}^{\prime}\right)$ a bien un sens. Berkovich a défini ([5], th. 7.1) une application $(x, t) \mapsto x_{t}$ de $\mathfrak{U}_{\eta}^{\prime} \times[0 ; 1]$ vers $\mathfrak{U}_{\eta}^{\prime}$ qui induit une rétraction de $\mathfrak{U}_{\eta}^{\prime}$ sur $S\left(\mathfrak{U}^{\prime}\right)$. Soit $P$ appartenant à $\Delta^{\prime}$. Chaque $g_{i}$ est inversible sur $\mathfrak{U}_{\eta}^{\prime}$. Les fonctions $t \mapsto\left|g_{i}\left(P_{t}\right)\right|$ et $t \mapsto\left|g_{i}^{-1}\left(P_{t}\right)\right|$ étant croissantes sur $[0 ; 1]$ pour tout $i$ (voir [5], énoncé (a) du th. 7.1) elles sont constantes. D'autre part si $\sum a_{I} \mathbf{T}^{I}$ est un polynôme à coefficients dans $k$ et si $t$ appartient à $[0 ; 1]$ on a :

$$
\left|\sum a_{I} \mathbf{g}\left(P_{t}\right)\right| \geq\left|\sum a_{I} \mathbf{g}(P)\right|
$$

(toujours d'après l'énoncé (a) du th. 7.1 de [5]) et

$$
\left|\sum a_{I} \mathbf{g}\left(P_{t}\right)\right| \leq \max _{I}\left|a_{I}\right| \cdot\left|\mathbf{g}\left(P_{t}\right)\right|=\max _{I}\left|a_{I}\right| \cdot|\mathbf{g}(P)|=\left|\sum a_{I} \mathbf{g}(P)\right| .
$$

On en déduit que

$$
\left|\sum a_{I} \mathbf{g}\left(P_{t}\right)\right|=\left|\sum a_{I} \mathbf{g}(P)\right|=\max _{I}\left|a_{I}\right| \cdot|\mathbf{g}(P)|
$$

et ce quel que soit le polynôme $\sum a_{I} \mathbf{T}^{I}$. En conséquence, $\varphi \circ \psi\left(P_{t}\right)$ appartient à $\mathbb{V}$ et est égal à $\varphi \circ \psi(P)$. Comme la dimension de $\mathfrak{U}_{\eta}^{\prime}$ sur $k$ est inférieure ou égale à $n$ et comme $\rho(\mathcal{H}(Q) / k)=n$ pour tout point $Q$ de $S_{k}^{n}(c f . \S 1.4)$, le lemme 1.9 assure que la fibre de $\varphi \circ \psi$ en tout point $Q$ de $\mathbb{V}$ est discrète. En conséquence $t \mapsto P_{t}$ est une application continue de $[0 ; 1]$ vers un ensemble discret et est donc constante. En particulier $P_{1}=P$ et $P$ appartient donc à $S\left(\mathfrak{U}^{\prime}\right)$.

3.25. Notons que $S\left(\mathfrak{U}^{\prime}\right)$ est a priori un espace ${\sqrt{\left|L^{*}\right|}}_{\mathbb{Q}^{-}}$linéaire par morceaux mais que $\sqrt{\left|L^{*}\right|}=\sqrt{\left|k^{*}\right|}$ puisque $L / k$ est finie; on peut donc le voir (ce que l'on fera) comme un espace $\sqrt{\left|k^{*}\right|}$-linéaire par morceaux. Les fonctions $g_{i}$ sont inversibles sur $U^{\prime} \simeq \mathfrak{U}_{\eta}^{\prime}$. D'après Berkovich (voir [1], th. 5.1.1, (5)), la restriction à $S\left(\mathfrak{U}^{\prime}\right)$ de chacune des fonctions $\left|g_{i}\right|$ est linéaire par morceaux. On peut donc écrire $S\left(\mathfrak{U}^{\prime}\right)$ comme une réunion finie $\bigcup_{s \in S} \mathcal{P}_{s}$ où $\mathcal{P}_{s}$ est pour 
tout $s$ un pseudo-polytope de dimension au plus $n$ et muni d'un système de coordonnées relativement auquel la famille des $\left|g_{i}\right|$ est affine. Soit $S^{\prime}$ l'ensemble des $s$ tels que la famille des $\left(\left|g_{i}\right|_{\mid \mathcal{P}_{s}}\right)$ constitue un système de coordonnées sur $\mathcal{P}_{s}$ (notons que dans ce cas $\mathcal{P}_{s}$ est de dimension $n$ ).

3.26. Proposition. - Le sous-ensemble $\Delta^{\prime}$ de $S\left(\mathfrak{U}^{\prime}\right)$ est la réunion des $\mathcal{P}_{s}$ pour $s$ dans $S^{\prime}$. C'est donc un sous-espace linéaire par morceaux de $S\left(\mathfrak{U}^{\prime}\right)$ et $\varphi \circ \psi_{\mid \Delta^{\prime}}: \Delta^{\prime} \rightarrow \mathbb{V}$ est linéaire par morceaux.

Démonstration. - On procède par double inclusion.

3.26.1. Soit $s$ appartenant à $S^{\prime}$ et soit $P$ un point de l'intérieur de $\mathcal{P}_{s}$. Supposons qu'il n'appartienne pas à $\Delta^{\prime}$. Il existe alors un polynôme $\sum a_{I} \mathbf{T}^{I}$ à coefficients dans $k$ tel que

$$
\left|\sum a_{I} \mathbf{g}^{I}(P)\right|<\max _{I}\left|a_{I}\right| \cdot|\mathbf{g}(P)|^{I} .
$$

En conséquence, $P$ possède un voisinage $\mathcal{Q}$ dans l'intérieur de $\mathcal{P}_{s}$ en tout point $Q$ duquel on a l'inégalité

$$
\left|\sum a_{I} \mathbf{g}^{I}(Q)\right|<\max _{I}\left|a_{I}\right| \cdot|\mathbf{g}(Q)|^{I} .
$$

Pour tout couple d'indices $(I, J)$ distincts avec $a_{I}$ et $a_{J}$ non nuls, appelons $\mathcal{Q}_{I, J}$ l'ensemble des points $Q$ de $\mathcal{Q}$ en lesquels $\left|a_{I}\right| \cdot\left|\mathbf{g}^{I}(Q)\right|=\left|a_{J}\right| \cdot\left|\mathbf{g}^{J}(Q)\right|$. On déduit de ce qui précède que $\mathcal{Q}$ est réunion des $\mathcal{Q}_{I, J}$. Chacun d'eux étant défini par une égalité entre fonctions affines l'un d'eux est nécessairement d'intérieur non vide dans $\mathcal{Q}$. Il existe donc deux indices $I$ et $J$ distincts tels que $a_{I}$ et $a_{J}$ soient non nuls et un pseudo-polytope $\mathcal{Q}_{0}$ de dimension $n$ inclus dans $\mathcal{P}_{s}$ sur lequel on a identiquement $\left|a_{I}\right| \cdot\left|\mathbf{g}^{I}\right|=\left|a_{J}\right| \cdot\left|\mathbf{g}^{J}\right|$. C'est contradictoire avec le fait que la famille des $\left(\left|g_{i}\right|_{\mid \mathcal{P}_{s}}\right)$ est un système de coordonnées sur $\mathcal{P}_{s}$. On en déduit que $P$ appartient à $\Delta^{\prime}$. En conséquence ce dernier contient la réunion des intérieurs des $\mathcal{P}_{s}$ pour $s$ parcourant $S^{\prime}$. Comme $\Delta^{\prime}$ est compact, il contient $\bigcup_{s \in S^{\prime}} \mathcal{P}_{s}$.

3.26.2. Montrons maintenant que $\Delta^{\prime} \subset \bigcup_{s \in S^{\prime}} \mathcal{P}_{s}$. Soit $P$ appartenant à $\Delta^{\prime}$. Alors $\varphi \circ \psi(P)$ appartient à $S_{k}^{n}$ et donc $\rho(\mathcal{H}(\varphi \circ \psi(P)) / k)=n(c f . \S 1.4)$; comme $\mathcal{Y}_{\eta}$ est de dimension $n$ on déduit du lemme 1.6 que $\rho(\mathcal{H}(\psi(P)) / k)=n$. Ceci entraîne, $\mathcal{Y}$ étant irréductible, que $\psi(P)$ n'appartient à aucun fermé Zariski strict de $\mathcal{Y}_{\eta}^{\text {an }}$. En particulier $\psi(P)$ appartient à $\mathcal{V}^{\text {an }}$. Dès lors $\psi$ est fini et plat en $P$, et il en va de même de $\psi_{\mid U^{\prime}}: U^{\prime} \rightarrow U$. Notons $\chi$ le morphisme

$$
\varphi \circ \psi_{\mid U^{\prime}}: U^{\prime} \longrightarrow V \text {. }
$$

Soit $\Omega$ un voisinage de $P$ dans $U^{\prime}$. D'après ce qui précède $\chi$ est fini et plat en $P$; en vertu de la proposition 3.2.7 de [3], l'ensemble $\chi(\Omega)$ contient alors un voisinage de $\chi(P)$ dans $V$.

Soit $s$ appartenant à $S-S^{\prime}$. Les fonctions $\left|g_{i}\right|_{\left.\right|_{\mathcal{P}}}$ sont affines et la famille des $\left|g_{i}\right|_{\mid \mathcal{P}_{s}}$ est par définition de $S^{\prime}$ de rang au plus $n-1$ modulo les constantes. 
Les $\left|g_{i}\right|_{\mid \mathbb{V}}$ constituent par ailleurs un système de coordonnées sur $\mathbb{V}$ et $\Delta^{\prime}$ n'est autre que $\chi^{-1}(\mathbb{V})$. On en déduit que

$$
\chi\left(\Delta^{\prime} \cap \bigcup_{s \in S-S^{\prime}} \mathcal{P}_{s}\right)
$$

est inclus dans la réunion d'un nombre fini de sous-espaces linéaires par morceaux de $\mathbb{V}$ de dimension au plus $n-1$ et en particulier ne contient aucun ouvert non vide de $\mathbb{V}$. Dès lors $\Omega \cap \Delta^{\prime}$ ne peut être inclus dans $\bigcup_{s \in S-S^{\prime}} \mathcal{P}_{s}$ et en conséquence rencontre $\bigcup_{s \in S^{\prime}} \mathcal{P}_{s}$.

Ceci est vrai quel que soit le voisinage $\Omega$ de $Q$. On en déduit que $Q$ est adhérent à $\bigcup_{s \in S^{\prime}} \mathcal{P}_{s}$ qui est un fermé de $S\left(\mathfrak{U}^{\prime}\right)$; finalement $Q$ appartient à $\bigcup_{s \in S^{\prime}} \mathcal{P}_{s}$ et donc $\Delta^{\prime} \subset \bigcup_{s \in S^{\prime}} \mathcal{P}_{s}$.

3.26.3. On a donc bien montré l'égalité $\Delta^{\prime}=\bigcup_{s \in S^{\prime}} \mathcal{P}_{s}$. Comme les $\left|g_{i}\right|_{\mathbb{V}}$ constituent un système de coordonnées sur $\mathbb{V}$ et comme la fonction $\left|g_{i}\right|_{\mid \Delta^{\prime}}$ est linéaire par morceaux pour tout $i$, la fonction $\varphi \circ \psi_{\mid \Delta^{\prime}}$ est linéaire par morceaux. La démonstration est terminée.

3.27. Comme $\mathcal{U}^{\prime}$ est l'image réciproque de $\mathcal{U}$, il est stable par l'action de $\mathbf{G}$, qui agit donc sur le schéma formel $\mathfrak{U}^{\prime}$ et en conséquence sur son squelette $S\left(\mathfrak{U}^{\prime}\right)$ (par automorphismes linéaires par morceaux). Le sous-espace linéaire par morceaux $\Delta^{\prime}$ de $S\left(\mathfrak{U}^{\prime}\right)$ est, par sa définition même, stable sous l'action de $\mathbf{G}$; il en va de même de $T^{\prime} \cap \Delta^{\prime}$, qui est un sous-espace linéaire par morceaux de $S\left(\mathfrak{U}^{\prime}\right)$ (voir Berkovich, [1], th. 6.3.1). On a vu ci-dessus que $\Delta^{\prime}$ était inclus dans $\psi^{-1}(\mathcal{V})^{\text {an }}$. La factorisation du morphisme $\psi^{-1}(\mathcal{V}) \rightarrow \mathcal{V}$ évoquée au $\S 3.23$ en induit une au niveau analytique, qui topologiquement correspond à un quotient par l'action de $\mathbf{G}$ suivi d'un homéomorphisme. En particulier le fermé $\Delta \cap T$ de $T$ est homéomorphe à $\left(\Delta^{\prime} \cap T^{\prime}\right) / \mathbf{G}$ et si on le munit de la structure linéaire par morceaux correspondante ([1], prop. 3.6.1), l'application $\varphi_{\mid \Delta \cap T}: \Delta \cap T \rightarrow \mathbb{V}$ est linéaire par morceaux.

La démonstration du théorème principal est terminée.

3.28. Fonctorialité. - Soit $n$ un entier. Donnons-nous un diagramme commutatif

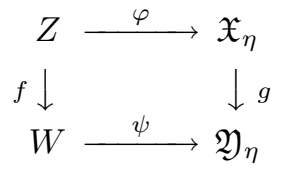

où $\mathfrak{X}$ et $\mathfrak{Y}$ sont deux $k^{0}$-schémas formels pluristables non dégénérés purement de dimension $n$, où $Z$ et $W$ sont deux espaces strictement $k$-analytiques topologiquement séparés de dimension au plus $n$, et où $g$ est induit par un morphisme étale $\mathfrak{X} \rightarrow \mathfrak{Y}$. Désignons par $\Delta$ (resp. $\Gamma$ ) l'ensemble $\varphi^{-1}(S(\mathfrak{X}))$ (resp. $\left.\psi^{-1}(S(\mathfrak{Y}))\right)$. On sait d'après Berkovich que $S(\mathfrak{X})$ est égal à $g^{-1}(S(\mathfrak{Y}))$, d'où il découle que $\Delta$ est égal à $f^{-1}(\Gamma)$, et que $g_{\mid S(\mathfrak{X})}: S(\mathfrak{X}) \rightarrow S(\mathfrak{Y})$ est linéaire par 
morceaux. Le théorème 3.1 affirme que l'on peut munir $\Delta$ (resp. $\Gamma$ ) d'une structure linéaire par morceaux compatible avec $\varphi$ (resp. avec $\psi$ ). Le corollaire 2.5 permet alors de conclure que $f$ induit une application linéaire par morceaux de $\Delta$ vers $\Gamma$.

3.29. Variation du corps résiduel. - Donnons-nous un diagramme $Z \rightarrow$ $\mathfrak{X}_{\eta}$ satisfaisant les hypothèses du théorème 3.1. Soit $\Delta$ l'espace linéaire par morceaux $\varphi^{-1}(S(\mathfrak{X}))$. Soit $\mathcal{R}$ un polynôme non nul à coefficients dans $k$ et soit $m$ un entier. Le sous-ensemble de $\Delta$ formé des points $P$ tels que $\mathcal{R}$ ait exactement $m$ facteurs irréductibles distincts sur le corps $\mathcal{H}(P)$ est alors un sous-espace linéaire par morceaux de $\Delta$ : il suffit en effet d'appliquer le résultat vu au $\S 3.28$ au diagramme

$$
Z \times{ }_{k} \mathcal{M}(k[t] / \mathcal{R}) \longrightarrow Z \longrightarrow \mathfrak{X}_{\eta} .
$$

Ce résultat généralise en partie une proposition de l'auteur valable pour les courbes (voir [8], prop. 2.3).

3.30. Polyèdre de variation. - Soit $n$ un entier et soit $Z$ un espace strictement $k$-analytique topologiquement séparé de dimension au plus $n$. Soit $\left(g_{1}, \ldots, g_{n}\right)$ une famille de $n$ fonctions inversibles sur $Z$ et $\Delta$ l'image réciproque de $S_{k}^{n}$ par la flèche $\varphi: Z \rightarrow\left(\mathbb{G}_{m, k}^{\text {an }}\right)^{n}$ induite par les $g_{i}$. On note $\lambda$ la flèche $Z \rightarrow\left(\mathbb{R}_{+}^{*}\right)^{n}$ qui envoie un point $P$ sur $\left(\left|g_{1}(P)\right|, \ldots,\left|g_{n}(P)\right|\right)$. On a vu au cours de la démonstration que $\Delta$ peut être muni d'une structure linéaire par morceaux compatible avec $\varphi(c f$. $\S 3.11)$. Soit $P$ appartenant au complémentaire de $\Delta$ dans $Z$. Le raisonnement suivi au $\S 3.26 .1$ montre qu'il existe un polynôme $\sum a_{I} \mathbf{T}^{I}$ en $n$ variables et un voisinage $U$ de $P$ dans $Z$ tel que pour tout point $Q$ de $U$ il existe deux indices $I$ et $J$ distincts vérifiant :

- $a_{I}$ et $a_{J}$ sont non nuls;

- $\left|a_{I}\right| \cdot\left|\mathbf{g}^{I}(Q)\right|=\left|a_{J}\right| \cdot\left|\mathbf{g}^{J}(Q)\right|$.

Le lieu de validité d'une égalité de la forme $\left|a_{I}\right| \cdot\left|\mathbf{g}^{I}\right|=\left|a_{J}\right| \cdot\left|\mathbf{g}^{J}\right|$ avec $I$ et $J$ distincts et $a_{I}$ et $a_{J}$ non nuls est un domaine analytique de $Z$; l'image par $\lambda$ de ce domaine analytique est par construction incluse dans un sous-espace affine de dimension $n-1$.

On déduit de tout ceci que si $V$ est un domaine strictement $k$-analytique compact de $Z$ ne rencontrant pas $\Delta$, le sous-ensemble $\lambda(V)$ de $\left(\mathbb{R}_{+}^{*}\right)^{n}$ est inclus dans une réunion finie d'espaces affines de dimension $n-1$; notons par ailleurs que $\lambda(V)$ est un sous-espace linéaire par morceaux de $\left(\mathbb{R}_{+}^{*}\right)^{n}(c f$. infra $)$. Ainsi $\Delta$ apparait comme une généralisation de la notion de polyèdre de variation introduite par l'auteur pour l'étude des fonctions analytiques sur une courbe (cf. [8], prop. 1.21).

À propos de la linéarité par morceaux de $\lambda(V)$. — Elle a été établie par Berkovich dans le cas où $V$ est quasi-algébrique, c'est-à-dire $G$-localement algébroïde (cf. [1], cor. 6.2.2). On va expliquer rapidement comment la prouver dans le 
cas général. On se ramène aussitôt au cas où $V$ est strictement $k$-affinoïde et intègre. On note $d$ sa dimension et l'on raisonne par récurrence sur $d$. Le cas $d=0$ est trivial. Supposons maintenant que $d$ est strictement positif, et que le résultat a été établi en dimension inférieure ou égale à $d-1$. Le lemme de normalisation de Nœther ( $c f$. [6], cor. 2 p. 228) assure l'existence d'un morphisme $\psi$ fini et surjectif de $V$ vers le polydisque unité de dimension $n$. Soit $P$ un point de $V$.

S'il n'est situé sur aucun fermé Zariski strict de $V$, un raisonnement analogue à celui tenu lors de la démonstration du lemme 1.9 permet de montrer que $\psi$ induit, entre un voisinage strictement $k$-affinoïde $U$ de $P$ et un voisinage strictement $k$-affinoïde $U^{\prime \prime}$ de $\psi(P)$ bien choisis, une flèche qui admet une factorisation de la forme $U \rightarrow U^{\prime} \rightarrow U^{\prime \prime}$ où $U \rightarrow U^{\prime}$ et $U^{\prime} \rightarrow U^{\prime \prime}$ sont deux revêtements finis et plats respectivement radiciel et étale. D'après le $\S 0.14$ il existe un entier $r$ strictement positif tel que pour tout $g$ appartenant à $\mathcal{O}(U)$ la fonction $g^{r}$ provienne de $\mathcal{O}\left(U^{\prime}\right)$. D'autre part $U^{\prime}$ est quasi-algébrique, puisque muni d'une flèche étale vers un espace quasi-algébrique. Le corollaire 6.2.2 de [1] implique alors que l'image de $U$ sous l'application $\left(\left|f_{1}\right|^{r}, \ldots,\left|f_{n}\right|^{r}\right)$ est un sousespace linéaire par morceaux de $\left(\mathbb{R}_{+}^{*}\right)^{n}$. Il en va de même de $\lambda(U)$, puisque $\left(t_{1}, \ldots, t_{n}\right) \mapsto\left(t_{1}^{r}, \ldots, t_{n}^{r}\right)$ est un automorphisme linéaire de $\left(\mathbb{R}_{+}^{*}\right)^{n}$.

Supposons maintenant que $P$ est situé sur un fermé Zariski strict $Y$ de $V$. D'après l'hypothèse de récurrence, $\lambda(Y)$ est un sous-espace linéaire par morceaux de $\left(\mathbb{R}_{+}^{*}\right)^{n}$. Posons $\Omega=\lambda^{-1}(\lambda(Y))$. Alors $\Omega$ est un domaine strictement $k$-analytique de $V$ qui contient $Y$ et qui est tel que $\lambda(\Omega)$ soit égal à $\lambda(Y)$, et soit donc un sous-espace linéaire par morceaux de $\left(\mathbb{R}_{+}^{*}\right)^{n}$. Comme $Y \rightarrow V$ est fini tout point de $Y$ appartient à Int $\Omega / V$, c'est-à-dire à l'intérieur topologique de $\Omega$ dans $V$. En conséquence $\Omega$ est un voisinage de $P$.

Finalement tout point du compact $V$ possède un voisinage compact dont l'image par $\lambda$ est un sous-espace linéaire par morceaux de $\left(\mathbb{R}_{+}^{*}\right)^{n}$. On en déduit que $\lambda(V)$ est un sous-espace linéaire par morceaux de $\left(\mathbb{R}_{+}^{*}\right)^{n}$.

\section{BIBLIOGRAPHIE}

[1] Berkovich (V.) - Smooth p-adic analytic spaces are locally contractible II, prépublication.

[2] - Spectral theory and analytic geometry over non-archimedean fields, Mathematical Surveys and Monographs, vol. 33, AMS, Providence, RI, 1990 .

[3] Étale cohomology for non-archimedean analytic spaces, Inst. Hautes Études Sci. Publ. Math., t. 78 (1993), pp. 5-161.

[4] _ Vanishing cycles for formal schemes, Invent. Math., t. 115 (1994), pp. 539-571.

BULlETIN DE LA SOCIÉtÉ MATHÉMATIQUE DE FRANCE 
[5] _ Smooth p-adic analytic spaces are locally contractible, Invent. Math., t. 137 (1999), pp. 1-84.

[6] Bosch (S.), Güntzer (U.) \& Remmert (R.) - Non-Archimedean analysis. A systematic approach to rigid analytic geometry, Grundl. Math. Wiss., vol. 261, Springer-Verlag, 1984.

[7] Bourbaki (N.) - Algèbre commutative, Hermann, 1964.

[8] Ducros (A.) - Cohomologie non ramifiée sur une courbe p-adique lisse, Compositio Math., t. 130 (2002), pp. 89-117. 